\title{
Sequential auctions with information about future goods
}

\author{
Robert Zeithammer
}

November 5, 2010

\begin{abstract}
When capacity-constrained bidders have information about a good sold in a future auction, they need to take the information into account in forming today's bids. The capacity constraint makes even otherwise unrelated goods substitutes and creates an equilibrium link between future competition and current bidding strategy. This paper proves the existence and uniqueness of a strictly monotone symmetric pure-strategy equilibrium under mild conditions on the population distribution of valuations, characterizes general properties of the equilibrium bidding strategy, and provides a simple technique for numerically approximating the bidding strategy for arbitrary valuation distributions. The key property of the equilibrium is that almost all bidders submit positive bids in the first stage, thereby ensuring trade with probability one. Even bidders who strongly prefer the second object submit a positive bid in the first auction, because losing the first auction is informative about the remaining competitors who also lost, and losing with a low bid indicates that these competitors are quite strong.
\end{abstract}

I would like to thank Birger Wernerfelt, Sergei Izmalkov, Sushil Bikhchandani, and seminar participants at UT Austin, CERGE-EI, CU Boulder, and UCLA for suggestions and help with this paper. Contact info: Robert Zeithammer, UCLA Anderson School of Management, 110 Westwood Plaza, Los Angeles, CA 90095. Email: rzeitham@ucla.edu 


\section{Introduction}

In many auction markets, similar goods are auctioned off in a pre-announced sequence: Governments and large firms auction off contracts for similar projects on a regular basis, often announcing future projects well in advance to allow bid-preparation. Estate auctions and auctions for property of retiring farmers are usually conducted as a sequence of auctions for the individual objects, with the entire set of auctioned goods available for inspection before the first auction begins. Consumers face sequential auctions with information about future goods on eBay, where sellers list upcoming auction-endings several days in advance. In all of the above situations, the individual auctioned goods are not necessarily identical, but they are substitutes to the bidders because of capacity constraints: a consumer buying a digital camera on eBay only has use for one camera, a construction firm can only fulfill a limited number of contracts given its technology, and a farmer may only need one tractor out of the several different tractors auctioned off in the sale of a retiring neighbor's machinery.

This paper models the sequential auctioning of two objects to unit-capacity bidders who may or may not be able to dispose of one object in case they end up with both. ${ }^{1}$ Each object is auctioned by a second-price sealed-bid auction with zero reserve. ${ }^{2}$ Each bidder has two nonnegative private valuations, one for each object, and the pairs of valuations are drawn independently across bidders from an arbitrary bivariate distribution with a bounded density and full support on a compact rectangle. This distributional assumption is general because not only can the two valuations be arbitrarily correlated, the distribution need not be symmetric in either support or shape, and it can even be discontinuous. Departing from most previous models of sequential auctions, each bidder knows her private value of the second unit before bidding on the first unit. The main finding of this paper is that a unique strictly monotone symmetric purestrategy Bayes-Nash equilibrium of the game exists. The equilibrium is strictly monotone in the sense that for every fixed valuation of the second object, first-auction bids strictly increase in the valuation of the first object.

In the proposed equilibrium, no bidders want to abstain from the first auction. This situation differs sharply from a best-response intuition about a rational bidder facing exogenous competition in the second stage: an exogenous second-stage competition would make a rational

\footnotetext{
${ }^{1}$ The basic model considers the case without disposal; the extension section analyzes the impact of free disposal.

${ }^{2}$ The extension section analyzes the impact of a first-stage reserve.
} 
bidder abstain from the first auction whenever her option value of losing (her expected secondstage surplus), exceeded her valuation of the first unit (her maximum first-stage surplus). No first-stage abstentions happen in the proposed equilibrium because the option value of the second auction is endogenous to first-stage bidding through the Bayesian equilibrium requirements. Let the two stages of the game be "today" and "tomorrow." The key tradeoff bidders face is between winning today and bidding tomorrow (today's winner exits the game because of the unit capacity assumption and no possibility of disposal). As in other models of sequential auctions for substitutes, the optimal bidding strategy solves this tradeoff by reducing today's bid to compensate for the opportunity cost of winning today, which is equal to a loser's expected surplus from participating in tomorrow's auction. The difference in the present model is that should I lose today, the competition I will face tomorrow depends on the bid-level at which I lose today: In equilibrium, all bidders reduce their bid today as a function of their values of tomorrow's object, so losing to a lower bid today makes higher competition tomorrow more likely. At the margin, I therefore need to assess the opportunity cost of winning today not only as a function of my valuation of tomorrow's object, but also as a function of the bid I submit today. In other words, losing at different bid-levels today is informative about the competition I can expect tomorrow. This paper analyzes this dependence and shows that it unravels any pure abstention strategy.

One way to understand the bidding incentives is to think of the first auction as an auction with a common value component arising from the fact that the opportunity cost of winning the first auction depends on the types of the other bidders. Since losing to a lower bid today makes higher competition tomorrow more likely, the common value component implies a "loser's curse" (Holt and Sherman 1994, Pesendorfer and Swinkels 1997) in that a failure to anticipate the informational content of winning makes one bid too low. Specifically, ignoring the information about tomorrow's competition contained in losing to a very low bid today would make some bidders abstain, only to be surprised tomorrow at the intensity of competition. This paper shows that taking this loser's curse into account is sufficient to rule out abstentions in a strictly monotone pure-strategy equilibrium.

The received theory of sequential auctions for substitutes focuses either on auctions of several identical units of a good (Milgrom and Weber 2000, Black and de Meza 1992, Katzman 1999) or on auctions of heterogeneous goods without information about future goods 
(Engelbrecht-Wiggans 1994, Jofre-Bonet and Pesendorfer 2003). When bidders demand more than one identical unit of the same good and have diminishing marginal utility, Black and de Meza (1992) and Katzman (1999) describe equilibria in which even multi-unit-demand bidders will make their first-stage bids contingent only on their valuations of the first unit. ${ }^{3}$ In contrast, the bidders in the proposed equilibrium always base their first-stage bids on both valuations. The proposed model generalizes Milgrom and Weber (2000) by separating the influence of information about future goods from the relationship between the private values of the two objects sold. Unlike in Milgrom and Weber's model, bidder types are two-dimensional here. Models with multi-dimensional types are rare in the auction literature because of the technical difficulties they pose. A seminal example is the model of Che and Gale (1998), who study the case of single-object auctions with each bidder facing a privately known budget constraint in addition to her valuation. As in Che and Gale (1998), the solution to the proposed model uses the concept of an isobid - a level curve of the two-dimensional bidding function.

The proposed model also generalizes Gale and Hausch (1994), who consider twodimensional information analogous to the present setting but solve the model only for two bidders. The extension to more bidders is non-trivial because it guarantees a non-degenerate second-stage competition to the losers of the first auction and leads to the endogeneity of this competition I describe above. The model predictions also differ qualitatively when more bidders are allowed: the probability of first-stage trade is less than one with only two bidders. This difference arises because the two-bidder model does not involve the loser's curse that makes bidders bid more aggressively in the first stage.

Finally, a version of the proposed model with free disposal, discussed in the Extensions section, is related and complementary to Burguet (2005). Burguet models a sequential ascending right-to-choose auction for two condominiums, one facing East and one facing West. In Burguet's model, each bidder wants only one condo and not all bidders prefer the same exposure. However, their private information is not truly two-dimensional because sorting the bidders according to their valuations of their preferred exposure automatically sorts them according to their valuations of their less-preferred exposure (the valuation of the less-preferred exposure is a publicly known monotonic function of the preferred-exposure valuation). This

\footnotetext{
${ }^{3}$ Their reasoning is also based on endogenous option value of the second auction: In equilibrium, bidders with diminishing marginal utility assume that should they lose the first auction, they will lose to another bidder with the same first-unit valuation, and that competitor will therefore win the second unit for sure.
} 
restriction is enough to make a sequential right-to-choose auction efficient - a result that does not necessarily hold in the more general setting considered here. Another model of sequential auctions with information about future goods without multi-dimensional types is Zeithammer (2006), who models auctions for movie DVDs on eBay as intertwined sequences of auctions, each sequence selling identical units of the same good (a particular movie-title) at varying timeintervals. This is analogous to Burguet's assumption with the value of the less-preferred exposure equal to zero.

In the terminology of Jehiel and Moldovanu (2000), the second auction is a particular example of a positive externality in a downstream interaction among the bidders. The expected surplus of a first-auction loser is the externality, it is positive by individual rationality, and it depends on the types of the other bidders. Unlike Jehiel and Moldovanu (2000) who analyze the first-stage response to a reduced-form externality with one-dimensional types, this paper models the externality in the form of another auction from structural primitives, and naturally considers two-dimensional types. As in Jehiel and Moldovanu (2000), the positive externality leads to a partial pooling by "low types" whenever the seller uses a reserve price. Another model with partial pooling at the reserve price due to a positive externality is the analysis of auctions with resale by Haile (2000). While the intuition for partial pooling by low types is the same here, the key difference with two-dimensional types lies in defining the meaning of "low type", i.e., in characterizing the continuum of valuation pairs that pool on the reserve in equilibrium.

Budish (2008) uses the computational procedure this paper proposes in order to assess the efficiency and distributional properties of the auction with information about future goods vis-àvis the efficient Vickrey-Clarke-Groves mechanism (VCG). He finds that under several different assumptions about the distribution of bidder valuations, the sequential auction is more than 99 percent efficient. Therefore, while monotonicity of the equilibrium does not imply efficiency as it would in an auction with single-dimensional bidders, the proposed equilibrium seems to be nearly efficient.

The paper is organized as follows: Section II presents the model, establishes existence and uniqueness of the equilibrium, and derives properties of the bidding function, and illustrates the model on a special case of the uniform distribution with support on $[0, K] \times[0,1]$.Section III discusses both possible extensions and barriers to further generalization. The most important extension is to the case of free disposal, a world in which the winner of the first object can still 
bid on the second object and dispose of the less-preferred object should she win both. The freedisposal case is technically analogous to the costly disposal situation of Section II, and the bidding function turns out to be similar in the uniform example. Another extension considers the equilibrium that arises when the seller uses a reserve inside the support of the first-object valuations: the bidding function changes relative to the no-reserve case in that the pricedistribution has a mass-point at the reserve level. Section IV concludes by interpreting the results within a broad framework of choice and sequential search and by highlighting both the contributions of the present work and potential avenues for future research.

\section{Model}

Two second-price sealed-bid auctions 1 and 2 are conducted in sequence, each auction selling one object with a zero reserve. $N>2$ risk-neutral bidders participate in the sequence of auctions. Each bidder has a single-object capacity in that she derives no value from a second object. ${ }^{4}$ Each bidder knows her private valuations of the two objects $\left(v_{1}, v_{2}\right)$ at the start of the game, and the two-dimensional bidder types are drawn independently across bidders from a distribution that satisfies:

Assumption 1: $\left(v_{1}, v_{2}\right)$ are distributed iid according to a distribution with a bounded density $f$ and full support on a closed and bounded rectangle $\left[L_{1}, H_{1}\right] \times\left[L_{2}, H_{2}\right] \subset \mathbf{R}_{+}^{2}$ where $H_{i}>L_{i} \geq 0$.

Correlation between the private values of the two objects captures the similarity of the two objects, and hence the degree to which they are substitutes in addition to the substitution resulting from the capacity constraint. ${ }^{5}$ The cumulative distribution of the valuations is denoted as $F(x, y)=\operatorname{Pr}\left(v_{1} \leq x \& v_{2} \leq y\right) . F\left(H_{1}, v_{2}\right)$ is therefore the marginal $c d f$ of $v_{2}$, and $F\left(v_{1}, H_{2}\right)$ is

\footnotetext{
${ }^{4}$ For example, the objects are two different cars, and each bidder only needs one car. Or the objects are procurement contracts, and each bidding firm can fulfill only one. Or the objects are tickets to two concurrent events.

${ }^{5}$ Consider two fundraiser dinners held simultaneously. When one is for the Republican Party and the other for the Democratic Party, the bidders' personal valuations are likely negatively correlated. In other words, the events are opposites and probably would not be substitutes without the capacity constraint. When one dinner is for the Republican Party and the other for a cause unrelated to politics, such as cancer research, then the valuations are uncorrelated: demand for one good is unrelated to demand for the other. Finally, when the two concurrent dinners are both for the Republican Party, the valuations are nearly perfectly correlated, and the two events would likely be substitutes even without the capacity constraint. Regardless of the cause each dinner supports, the two events are substitutes in the proposed model because of the capacity constraints: each participant can only attend one of them.
} 
the marginal $c d f$ of $v_{1}$. Extending the notation, define the cumulative distribution function under a continuous curve $\psi:\left[L_{2}, H_{2}\right] \rightarrow\left[L_{1}, H_{1}\right]$ as $F(\psi, y) \equiv \int_{L_{2}}^{y} \int_{L_{1}}^{\psi\left(v_{2}\right)} f\left(v_{1}, v_{2}\right) d v_{1} d v_{2}$. Note that the generality of the distributional assumption makes it without loss of generality to assume that the two auctions follow promptly after each other, so there is no discounting of the second auction's outcome.

While the distributional assumption is necessary for existence of a strictly monotone pure strategy equilibrium, the following assumption about the utility of winning both objects is merely convenient and will be relaxed in the Extensions section: Disposal or resale of a purchased object is costly enough that the winner of the first auction does not bid again. ${ }^{6}$

I restrict attention to strictly monotone symmetric pure-strategy equilibria, where "monotone" means that the first-auction bids increase in $v_{1}$ for every fixed $v_{2}$, and "strictly" rules out pooling on the same bid-level for a range of different $v_{1}$ s. The second stage has a dominant strategy to bid valuation $v_{2}$ because it is a single second-price sealed-bid auction. The dominant strategy ensures that the information disclosure in the end of the first stage is irrelevant: the second-stage bidding is the same regardless of whether the bidders learn nothing, only the price, or all the bids of the first stage. This is the principal simplification relative to the first-price sealed-bid format, where disclosure of first-stage bids matters (as demonstrated for the case of two bidders by Reiß and Schöndube 2008).

The main goal of this paper is characterizing first-stage bidding given the simple second stage. Potential participation in the second stage yields a non-negative expected surplus to the losers of the first stage. The expected surplus of a focal first-stage loser depends obviously on her $v_{2}$, and less obviously on the winning bid in the first stage, hereafter denoted $c_{1}$. I denote this expected surplus function as $S\left(v_{2}, c_{1}\right)$. $S$ depends on $c_{1}$ because competitors' first-stage bids depend on their respective $v_{2} \mathrm{~s}$, and so losing the first stage to a particular $c_{1}$ contains information about $v_{2}$ s of the remaining competitors (who also lost to that same $c_{1}$ ). Note that this information does not somehow impact second-stage bidding (which is fixed by the dominant strategy

\footnotetext{
${ }^{6}$ A demand-assumption equivalent to costly disposal would be that the value of the second object contingent on owning the first object is zero. Thus, the winner of the first car cannot sell or even throw away the first car in order to free up his garage for the second car. Analogously, the firm receiving the first contract must fulfill it before bidding again, and sub-contracting is not feasible. Or there simply is no time to turn around and re-sell won tickets to this evening's events. The Extensions section will explore the impact of free disposal.
} 
described in the previous paragraph). Instead, the information about remaining competitors enters the first-stage bidding through marginal analysis in a Bayesian equilibrium: the focal bidder assumes that should she lose the first auction, she would lose in a tie to a $c_{1}$ equal to her own bid. In other words, the focal bidder assumes she is pivotal to the outcome of the first stage.

It will be very convenient throughout this paper to describe the first-stage bidding function $b_{1}\left(v_{1}, v_{2}\right)$ as a set of isobids - level curves of $b_{1}(\cdot)$ :

Definition: isobid for bid-level $\boldsymbol{\beta}$ is a function $I\left(v_{2} \mid \beta\right):\left[L_{2}, H_{2}\right] \rightarrow\left[L_{1}, H_{1}\right]$ such that

$$
b_{1}\left(I\left(v_{2} \mid \beta\right), v_{2}\right)=\beta \text {. }
$$

I look for an intuitive monotone pure-strategy equilibrium in which each bidder bids their value of the first object net of her expected surplus of losing given that $c_{1}=v_{2}: b_{1}\left(v_{1}, v_{2}\right)=v_{1}-S\left(v_{2}, b_{1}\right)$. Because of the truth-revealing pivotal nature of the second-price auction, this form of the bidding function is without loss of generality in a symmetric strictly monotone pure-strategy equilibrium: given any $b_{1}\left(v_{1}, v_{2}\right)$ in such an equilibrium, is must be the case that $\tilde{S}\left(v_{2}, b_{1}\right)=v_{1}-b_{1}\left(v_{1}, v_{2}\right)$ is in fact the expected surplus of a first-auction loser. If not, then there is a profitable deviation. Isobids of such a bidding function have the following convenient form:

$$
I\left(v_{2} \mid \beta\right)=\beta+S\left(v_{2}, \beta\right)
$$

The relationship between the isobid $I$ and the expected surplus function $S$ shown in equation (1) leads to a useful equilibrium restrictions on isobids, because the actual expected surplus on the RHS of equation (1) in turn depends on the isobids it implies on the LHS. Specifically,

Proposition 1: When a set of curves $\left\{I\left(v_{2} \mid c_{1}\right)\right\}$ for $c_{1} \in\left[L_{1}, H_{1}\right]$ is a set of isobids of a strictly monotone symmetric pure-strategy Bayesian Nash equilibrium $b_{1}\left(v_{1}, v_{2}\right)=v_{1}-S\left(v_{2}, b_{1}\right)$, then each curve must satisfy :

$$
I\left(v_{2} \mid c_{1}\right)=\boldsymbol{T}\left(I \mid c_{1}\right)\left(v_{2}\right)=c_{1}+\int_{L_{2}}^{v_{2}}\left(\frac{F\left[I\left(\cdot \mid c_{1}\right), x\right]}{F\left[I\left(\cdot \mid c_{1}\right), H_{2}\right]}\right)^{N-2} d x
$$


I include the proof of Proposition 1 in the main text because it is instructive about the nature of equilibrium in this game. Consider a single focal bidder in the first auction. Suppose all of her competitors happen to be bidding according to $b_{1}\left(v_{1}, v_{2}\right)=v_{1}-S\left(v_{2}, b_{1}\left(v_{1}, v_{2}\right)\right)$. When the highest competing bid in the first stage is $c_{1} \geq L_{1}>0$, the focal bidder can assume that there will be trade in the first period, and so losing to $c_{1}$ in the first stage means there will be $N-2$ surviving competitors in the second stage. All surviving competitors will also have lost to $c_{1}$ in the first stage, so their $\left(v_{1}, v_{2}\right)$ types are selected such that $b_{1}\left(v_{1}, v_{2}\right)<c_{1}$ (the competitor who bids exactly $c_{1}$ wins the first auction and exits the game). Since $b_{1}\left(v_{1}, v_{2}\right)$ is increasing in $v_{1}$, the isobids $I\left(v_{2} \mid \beta\right)$ defined in equation (1) are increasing in $\beta$, and so $b_{1}\left(v_{1}, v_{2}\right)<c_{1}$ means $v_{1}<I\left(v_{2} \mid c_{1}\right)$, i.e., the surviving competitors have $\left(v_{1}, v_{2}\right)$ below the $I\left(v_{2} \mid c_{1}\right)$ isobid. In the second stage, all bidders bid their valuation, and so the highest competing bid $c_{2}$ is the highest of $N-2$ iid draws from the distribution of $v_{2}$ such that $v_{1}<I\left(v_{2} \mid c_{1}\right)$. By Bayes Theorem, each such $v_{2}$ is distributed according to a distribution function $G_{2}\left(c_{2} \mid c_{1}\right)$ defined by the ratio of the $f$ probability mass below $I\left(v_{2} \mid c_{1}\right)$ and to the left of $c_{2}$, and the entire probability mass under the isobid $I\left(v_{2} \mid c_{1}\right):$

$$
G_{2}\left(c_{2} \mid c_{1}\right)=\operatorname{Pr}^{N-2}\left(v_{2} \leq c_{2} \mid b_{1}\left(v_{1}, v_{2}\right) \leq c_{1}\right)=\left(\frac{F\left[I\left(\cdot \mid c_{1}\right), c_{2}\right]}{F\left[I\left(\cdot \mid c_{1}\right), H_{2}\right]}\right)^{N-2} \stackrel{(F i g 1)}{=}\left(\frac{\int_{X} f(w) d w}{\int_{X+Y} f(w) d w}\right)^{N-2}
$$

where $\mathrm{X}$ and $\mathrm{Y}$ are the pertinent areas under the isobid illustrated in Figure 1. A set of distributions $G_{2}\left(c_{2} \mid c_{1}\right)$ for every $c_{1}$ implies the following expected surplus function:

$$
S\left(v_{2}, c_{1}\right)=\int_{L_{2}}^{v_{2}}\left(v_{2}-c_{2}\right) d G_{2}\left(c_{2} \mid c_{1}\right)=\int_{L_{2}}^{v_{2}} G_{2}\left(c_{2} \mid c_{1}\right) d c_{2}=\int_{L_{2}}^{v_{2}}\left(\frac{F\left[I\left(\cdot \mid c_{1}\right), c_{2}\right]}{F\left[I\left(\cdot \mid c_{1}\right), H_{2}\right]}\right)^{N-2} d c_{2}
$$

where the second equality follows from integration by $\operatorname{parts}^{7}$, and the last equality follows from equation (3). Plugging equation (4) into equation (1) completes the proof. QED Proposition 1.

\footnotetext{
${ }^{7}$ This is standard: the expected surplus in a standard auction is the integrated probability of winning (Krishna 2002).
} 


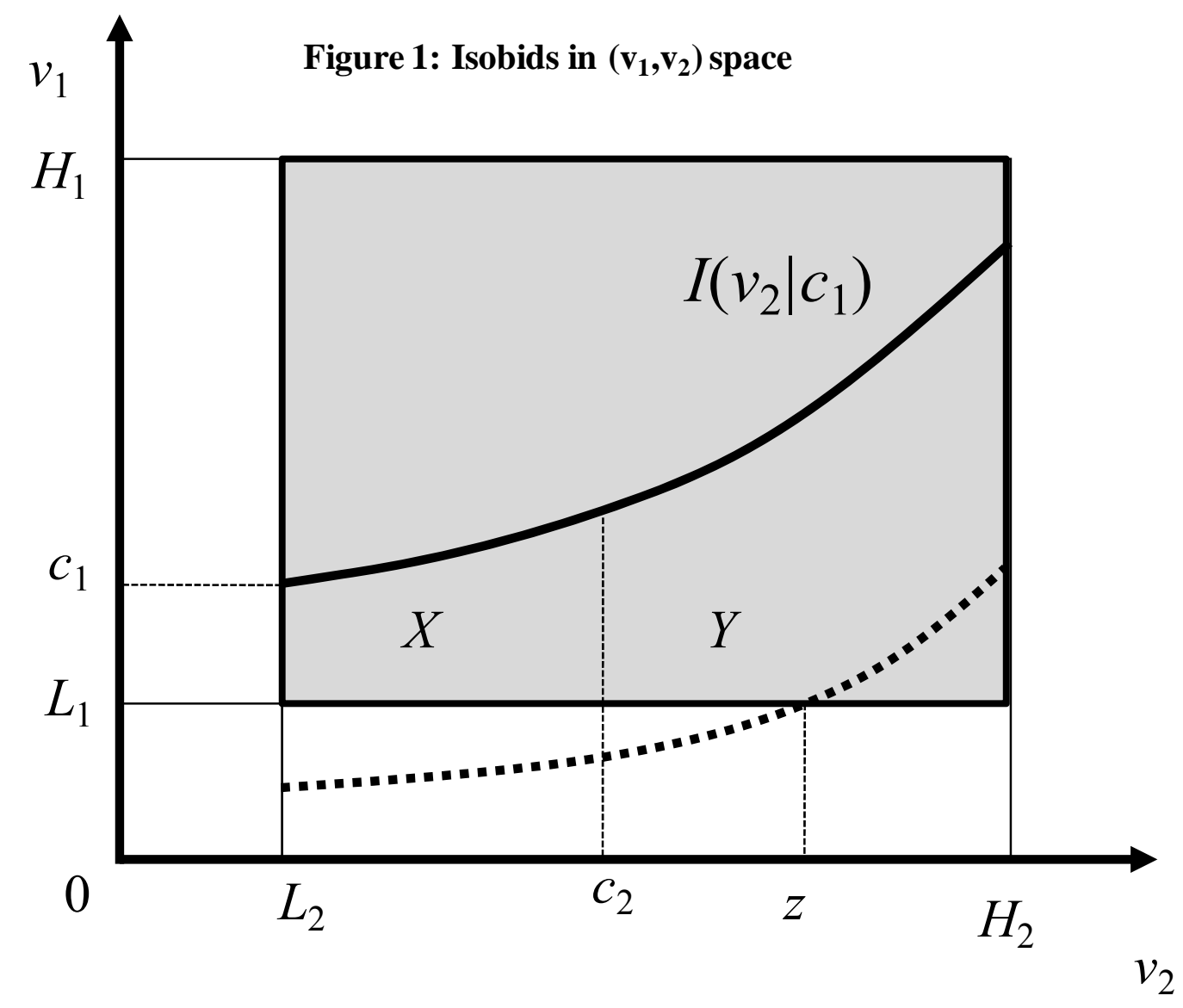

The intuition for Proposition 1 is that a particular set of isobids implies a belief about the expected surplus, and this belief in turn implies another set of isobids via equation (1) whenever the underlying bidding function $b_{1}\left(v_{1}, v_{2}\right)$ is increasing in $v_{1}$. In a Bayesian equilibrium, the belief must be correct. The benefit of $b_{1}\left(v_{1}, v_{2}\right)$ strictly monotone in $v_{1}$ is that the isobids are increasing in the bid-level, and so bidding below any particular bid-level $\beta$ implies having a set of valuations $\left(v_{1}, v_{2}\right)$ below the isobid for bid-level $\beta$. This is precisely the sense in which losing to a different high bidder in the first stage is informative about the $v_{2}$. In equation (2), $\boldsymbol{T}(J \mid \beta)$ is a mapping on the space of functions defined by $\boldsymbol{T}(J \mid \beta)\left(v_{2}\right)=\beta+\int_{0}^{v_{2}}\left(\frac{F[J, x]}{F\left[J, H_{2}\right]}\right)^{N-2} d x$, and equation (2) restricts any equilibrium $I\left(v_{2} \mid c_{1}\right)$ to be a fixed point of $\boldsymbol{T}\left(\cdot \mid c_{1}\right)$. Specifically, any equilibrium with $b_{1}\left(v_{1}, v_{2}\right)$ is increasing in $v_{1}$ must satisfy an integral equation that links the curvature of $I$ to probability mass under it. To see the relationship between the curvature and area 
underneath, multiply equation (2) by $F\left[I\left(\cdot \mid c_{1}\right), H_{2}\right]$ and differentiate the result twice (suppressing $c_{1}$ for clarity):

$I^{\prime \prime}\left(v_{2}\right) F^{N-2}\left(I, H_{2}\right)=(N-2) F^{N-3}\left(I, v_{2}\right) \int_{L_{1}}^{I\left(v_{2}\right)} f\left(v_{1}, v_{2}\right) d v_{1}$ such that $I\left(L_{2}\right)=c_{1}, I^{\prime}\left(L_{2}\right)=0$.

The alternative expression (2') is useful whenever $F\left[I\left(\cdot \mid c_{1}\right), H_{2}\right]=0$, and equation (2) is thus not defined.

I now construct a symmetric pure-strategy Bayesian Nash equilibrium with $b_{1}\left(v_{1}, v_{2}\right)$ increasing in $v_{1}$ in three steps that can be outlined as follows:

1) The full support and boundedness of $f$ imply that for every $\beta \in\left(L_{1}, H_{1}\right]$, a unique function $I$ exists that satisfies equation (2). This function is a candidate for an isobid curve $I\left(v_{2} \mid \beta\right)$.

2) The set of candidate isobids $I\left(v_{2} \mid \beta\right)$ for all $\beta \in\left(L_{1}, H_{1}\right]$ implies a candidate surplus function $S\left(v_{2}, c_{1}\right)=I\left(v_{2} \mid c_{1}\right)-c_{1}$. The full support and boundedness of $f$, together with the properties of the candidate isobids implied by equation (2), ensure that $S\left(v_{2}, c_{1}\right)$ is regular. Regularity is defined as follows:

Definition: A function $S\left(v_{2}, c_{1}\right)$ on $\left[L_{2}, H_{2}\right] \times\left[L_{1}, H_{1}\right]$ is regular when it satisfies all of the following conditions:

A) For every $v_{2} \in\left[L_{2}, H_{2}\right], S$ does not decrease in $c_{1}$ faster than unity:

$$
\forall c<d \in\left(L_{1}, H_{1}\right), S\left(v_{2}, d\right)-S\left(v_{2}, c\right)>(-1)(d-c) .
$$

B) $S$ is continuous in $c_{1}$ on $c_{1} \in\left[L_{1}, H_{1}\right]$.

C) For every $v_{2} \in\left[L_{2}, H_{2}\right], S\left(v_{2}, L_{1}\right)=0$.

3) The first stage has a unique best response function $b_{1}\left(v_{1}, v_{2}\right)$ to any regular expected surplus function $S\left(v_{2}, c_{1}\right)$ arising from second-stage bidding. The best response is defined implicitly by $b_{1}\left(v_{1}, v_{2}\right)=v_{1}-S\left(v_{2}, b_{1}\left(v_{1}, v_{2}\right)\right)$ and regularity implies that $b_{1}\left(v_{1}, v_{2}\right)$ is increasing in $v_{1}$, so the candidate isobids from Step 1 are indeed equilibrium isobids. 


\section{Step 1: Existence and uniqueness of candidate isobids}

The equilibrium condition in equation (2) immediately rules out an intersection between a candidate isobid and the lower boundary of the support rectangle, so a symmetric pure-strategy equilibrium with bidding below $L_{1}$ cannot exist:

Proposition 2 (No candidate isobids for bidding below $L_{1}$ ): There does not exist any candidate equilibrium isobid $I\left(v_{2} \mid \beta\right)$ for $\beta<L_{1}$ such that $I\left(v_{2} \mid c_{1}\right)>L_{1}$ for some $v_{2} \in\left[L_{2}, H_{2}\right]$.

Please see the appendix for detailed proofs of all remaining propositions. Proposition 2 shows that the dashed line in Figure 1 cannot satisfy the equilibrium condition. The reason is that the slope of a candidate isobid curve at any given $v_{2}$ is proportional to the probability mass under the curve and left of $v_{2}$, but there is no probability mass near the dashed curve's intercept, and so the curve cannot have positive slope there. From a bidder's perspective, consider the bidder $\left(L_{1}, z\right)$ at the intersection of the dashed curve and the lower boundary of the valuation support: at the margin (under the curve), she is guaranteed to lose the second auction by encountering only stronger competitors, and so her best response to the dashed curve is to not shade her first-stage bid below her valuation $v_{1}=L_{1}$, which is a higher first-stage bid level than the bid-level corresponding to the dashed curve.

Symmetric pure-strategy equilibrium considerations alone thus restrict attention to firstround bids at or above the lower bound of the support of $v_{1}$. For bid-levels above $L_{1}$, the candidate-isobid situation is surprisingly orderly given the mild distributional assumptions:

Proposition 3 (Existence and uniqueness of candidate equilibrium isobids) : For each $c_{1} \in\left(L_{1}, H_{1}\right]$, there exists a unique nondecreasing 1-Lipschitz function $I\left(v_{2} \mid c_{1}\right):\left[L_{2}, H_{2}\right] \rightarrow\left[c_{1}, c_{1}+H_{2}-L_{2}\right]$ with $I\left(L_{2} \mid c_{1}\right)=c_{1}$ such that $I\left(L_{2} \mid c_{1}\right)=\boldsymbol{T}\left[I\left(L_{2} \mid c_{1}\right) \mid c_{1}\right]$.

The proof relies on showing that a $K$-times repetition of the mapping $\boldsymbol{T}$ defined in equation (2) is a contraction map on the closed metric space of all nondecreasing 1-Lipschitz functions on the 
support of $v_{2}$ with $I\left(L_{2}\right)=c_{1}$ (under the supremum metric). Therefore, the Banach Fixed point theorem implies existence and uniqueness of a fixed point of $\boldsymbol{T}^{8}$

Proposition 3 plays an important role in reducing the dimensionality of the problem of finding the two-dimensional equilibrium-function $b_{1}\left(v_{1}, v_{2}\right)$ : for a given level of $b_{1}=\beta$, the candidate equilibrium isobid $I\left(v_{2} \mid \beta\right)$ is only a one-dimensional function that depends on $f$ and does not depend on other candidate isobids.

\section{Step 2: Regularity of the expected surplus function implied by candidate isobids}

The full set of candidate isobids $\left\{I\left(v_{2} \mid c_{1}\right)\right\}_{c_{1}>L_{1}}^{H_{1}}$ from Proposition 3 implies a unique candidate for the expected surplus function defined on $\left[L_{2}, H_{2}\right] \times\left(L_{1}, H_{1}\right]$ :

$$
S\left(v_{2}, c_{1}\right)=I\left(v_{2} \mid c_{1}\right)-c_{1}
$$

Extend the surplus function to the entire closed support rectangle by defining it as a limit $S\left(v_{2}, L_{1}\right)=\lim _{c \rightarrow L_{1}+} I\left(v_{2} \mid c\right)-L_{1}$. The properties of candidate isobids translate immediately into the properties of $S$ as a function of $v_{2}$. The analysis that follows uses some of these properties extensively, so I state them first:

Proposition 4: The candidate surplus function $S\left(v_{2}, c_{1}\right) \geq 0, S$ is non-negative, twice partially differentiable in $v_{2}$, non-decreasing in $v_{2}$, 1-Lipschitz in $v_{2}$, and convex in $v_{2}$.

Proposition 4 is immediate from the fact that $S$ is an integrated cumulative distribution function:

$$
S\left(v_{2}, c_{1}\right)=\int_{L_{2}}^{v_{2}} G_{2}\left(c_{2} \mid c_{1}\right) d c_{2} \geq 0 \Rightarrow 1 \geq \frac{\partial S}{\partial v_{2}}=G_{2}\left(v_{2} \mid c_{1}\right) \geq 0 \Rightarrow \frac{\partial^{2} S}{\partial v_{2}^{2}} \propto \frac{\int_{L_{1}}^{I\left(v_{2} \mid c_{1}\right)} f\left(v_{1}, v_{2}\right) d v_{1}}{F\left[I\left(\cdot \mid c_{1}\right), H_{2}\right]} \geq 0
$$

where all inequalities are strict when $v_{2}>L_{2}$. The intuition for Proposition 4 is straightforward: The expected surplus increases in the valuation $v_{2}$ because a higher valuation makes winning

\footnotetext{
${ }^{8}$ Mere existence follows from the Schauder fixed-point theorem because the metric space of functions in Proposition 3 is compact: Arzela-Ascoli Theorem implies that a set of continuous real functions on a compact interval is compact when it is closed, bounded, and equicontinuous. The first two conditions hold by definition, and the 1-Lipschitz continuity of the image of $\boldsymbol{T}(I)$ implies equicontinuity.
} 
more likely and also increases the actual surplus conditional on winning. Since these two atleast-linearly increasing components effectively multiply to produce the expected surplus, the convexity results. Since increasing $v_{2}$ by a small amount can increase the expected future surplus at most by that amount (and that only in the case when future prices are guaranteed to be below $v_{2}$ ), the slope of $S$ in $v_{2}$ is bounded above by unity. The regularity argument builds on Proposition 4 by showing that $S$ is not only well-behaved as a function of $v_{2}$, but also as a function of $c_{1}$. I demonstrate the three regularity conditions $\mathrm{A}, \mathrm{B}$, and $\mathrm{C}$ in turn.

To show that $S$ does not decrease in $c_{1}$ faster than unity, it is enough to show that two candidates for equilibrium isobids cannot cross or even touch each other, i.e. that $I\left(v_{2}, c_{1}\right)$ is strictly increasing in both arguments:

Proposition 5 (Part A of regularity): For every $c, d \in\left(L_{1}, H_{1}\right): d>c \Rightarrow I(v \mid d)>I(v \mid c)$ for every $v \in\left[L_{2}, H_{2}\right]$

The result of Proposition 5 is implied by the full support assumption together with the fact that a slope of any candidate for equilibrium isobid $I(v \mid c)$ at $w$ is $G_{2}(w \mid c)$, which is in turn a ratio of probability mass under $I(v \mid c)$ and left of $w$ and the probability mass under the entire $I(v \mid c)$. This relation between the candidate isobid and its slope implies that when $d>c$, the $I(v \mid c)$ that starts out below $I(v \mid d)$ at $v_{2}=L_{2}$ cannot have the required higher slope at a potential intersection point with the higher $I(v \mid d)$. Interestingly, even a tangency is ruled out, so $I\left(v_{2}, c_{1}\right)$ is strictly increasing in both arguments, and $S\left(v_{2}, c_{1}\right)=I\left(v_{2} \mid c_{1}\right)-c_{1}$ thus cannot decrease in $c_{1}$ even weakly faster than unity.

The second (B) part of regularity (continuity in $c_{1}$ ) follows from uniqueness of candidate isobids and the global bound on their slopes.

Proposition 6 (Part $B$ of regularity except for continuity at $L_{1}$ ): The candidate equilibrium surplus function $S\left(v_{2}, c_{1}\right)$ is continuous in $c_{1}$ at all $c_{1} \in\left(L_{1}, H_{1}\right]$.

The argument proceeds as follows: Together with compact support of the candidate isobids, the global bound on their slopes guarantees that as $\varepsilon$ approaches zero, $I\left(v_{2} \mid c_{1}+\varepsilon\right)$ converges 
uniformly to a nondecreasing 1-Lipschitz function $I_{+}:\left[L_{2}, H_{2}\right] \rightarrow\left[c_{1}, c_{1}+H_{2}-L_{2}\right]$ with $I_{+}\left(L_{2}\right)=c_{1}$. Together with the global bound on $f$, uniform convergence of the candidate isobids then implies that when $c_{1} \in\left(L_{1}, H_{1}\right], I_{+}$satisfies the equilibrium relation in equation (2), so $I_{+}$must be $I\left(v_{2} \mid c_{1}\right)$ because a unique function exists that satisfies that relation. The argument for $I\left(v_{2} \mid c_{1}-\varepsilon\right)$ converging uniformly to the $c_{1}$ candidate isobid from below is analogous, so continuity results.

The final part of regularity is the most striking: the candidate equilibrium surplus vanishes as the bid-level $c_{1}$ approaches the lower bound of the support $L_{1}$, even when the $v_{2}$ is large relative to the competition, for example, when $v_{2}=H_{2}$. Given equation (5), showing that candidate isobids $I\left(v_{2} \mid c_{1}\right)$ approach the constant function $I\left(v_{2} \mid L_{1}\right)=L_{1}$ as $c_{1}$ approaches $L_{1}$ is enough.

Proposition 7 (Part $\boldsymbol{C}$ of regularity and continuity at $\boldsymbol{L}_{\mathbf{1}}$ ): As $c_{1}$ approaches $L_{1}, \quad I\left(v_{2} \mid c_{1}\right)$ converge uniformly to $I\left(v_{2} \mid L_{1}\right)=L_{1}$, and so $S\left(v_{2}, c_{1}\right)$ converges to $S\left(v_{2}, L_{1}\right)=0$.

Note that by defining $I\left(v_{2} \mid L_{1}\right)$ as a limit, Proposition 7 extends the candidate surplus function $S\left(v_{2}, c_{1}\right)=I\left(v_{2} \mid c_{1}\right)-c_{1}$ to be continuous on the entire closed support $\left[L_{2}, H_{2}\right] \times\left[L_{1}, H_{1}\right]$. The equilibrium condition (2) is not defined for the function $I\left(v_{2} \mid L_{1}\right)=L_{1}$ because the mass below it is zero, but the equivalent equilibrium condition (2') holds, and so $b_{1}\left(L_{1}, v_{2}\right)=L_{1}$ is a candidate for an equilibrium isobid. Proposition 7 essentially shows that $b_{1}\left(L_{1}, v_{2}\right)=L_{1}$ is a unique candidate, and that higher $I\left(v_{2} \mid c_{1}\right)$ converge to it.

A useful metaphor helps explain the intuition for Proposition 7: Imagine a horizontally oriented zipper, with the lower side of the zipper sewn to a constant function $\left[L_{2}, H_{2}\right] \rightarrow L_{1}$, and the upper side representing the candidate isobid for the $L_{1}$ level. The zipper is stitched closed at the left end $\left(L_{2}, L_{1}\right)$ by definition of a candidate isobid. The intuition for candidate isobids of smaller and smaller bid-levels converging to the constant function $L_{1}$ is that constructing the $I\left(v_{2} \mid L_{1}\right)$ starting at $\left(L_{2}, L_{1}\right)$ is like closing the zipper from left to right: The 
slope of $I$ at $v_{2}=L_{2}$ is zero (true for all candidate isobids), and so is its curvature (not true for any other candidate isobid) because there is no mass below $L_{1}$. Starting with the zipper open, i.e. with a mass somewhere under the candidate isobid, the zero curvature means that the mass cannot be near $\left(L_{2}, L_{1}\right)$. This in turn means that the zipper is already closed in a neighborhood of $v_{2}=L_{2}$, and the same zero-curvature argument as for $v_{2}=L_{2}$ can be applied to some larger $v_{2}$ for which the zipper is still closed, i.e. for which $I\left(v_{2} \mid L_{1}\right)=L_{1}$.

From the perspective of the bidders, an $L_{1}$ candidate isobid $I\left(v_{2} \mid L_{1}\right)$ with a positive mass underneath unravels as follows: Suppose a $\operatorname{bidder}\left(v_{1}, v_{2}\right)$ with $v_{1}>L_{1}$ is on the $I\left(v_{2} \mid L_{1}\right)$ isobid line. In expecting a positive expected surplus should she lose, the bidder is relying on other bidders $\left(\tilde{v}_{1}, \tilde{v}_{2}\right)$ with smaller but still non-minimum valuations $L_{i}<\tilde{v}_{i}<v_{i}$ to also bid $L_{1}$ ( $L_{i}<\tilde{v}_{i}$ is critical for a positive mass of such competitors). In a symmetric equilibrium, those bidders $\left(\tilde{v}_{1}, \tilde{v}_{2}\right)$ rely on other bidders with yet smaller but still non-minimum valuations to also bid $L_{1}$, all the way down to bidders arbitrarily near the point $\left(L_{2}, L_{1}\right)$. But bidders on $I\left(v_{2} \mid L_{1}\right)$ sufficiently close to $\left(L_{2}, L_{1}\right)$ realize they will lose the second auction almost surely because the probability mass under $I\left(v_{2} \mid L_{1}\right)$ and left of a small $v_{2}$ is zero (since an equilibrium $I$ must have a slope and curvature of zero at $L_{2}$ ). Therefore, the cascade unravels, the $\left(\tilde{v}_{1}, \tilde{v}_{2}\right) \approx\left(L_{2}, L_{1}\right)$ competitors bid a small amount strictly greater than $L_{1}$, and the focal $\operatorname{bidder}\left(v_{1}, v_{2}\right)$ thus also bids strictly more than $L_{1}$. This unraveling corrects for the loser's curse that would result from bidding $L_{1}$.

\section{Step 3: Candidate isobids are the best response to the candidate expected surplus function}

Consider a single focal bidder, and let $c_{1}$ be the highest first-stage competitive bid she faces, that is, the maximum first bid of her $N-1$ first-stage competitors. Suppose the focal bidder believes $c_{1}$ to be distributed according to some distribution $G_{1}$ on $\left[0, H_{1}\right]$, and believes that $S$ captures the possible second-stage payoffs. The focal bidder solves the following problem in the first auction: 


$$
b_{1}\left(v_{1}, v_{2}\right)=\underset{\beta}{\arg \max }\left\{\int_{0}^{\beta}\left(v_{1}-c_{1}\right) d G_{1}\left(c_{1}\right)+\int_{\beta}^{\infty} S\left(v_{2}, c_{1}\right) d G_{1}\left(c_{1}\right)\right\}
$$

When $S$ is regular, the following first-order condition characterizes the best response to $S$ :

$$
\text { FOC }: b_{1}\left(v_{1}, v_{2}\right)=\beta \text { such that } \beta=v_{1}-S\left(v_{2}, \beta\right)
$$

Regularity of $S$ ensures both concavity of the focal bidder's objective function at the $F O C$ and sub-optimality of corner solutions (i.e., suboptimality of bidding $L_{1}$ or $H_{1}$ ). In addition, regularity implies that the implicit function $b_{1}\left(v_{1}, v_{2}\right)$ is well-defined and its image is restricted to $b_{1} \geq L_{1}$. Finally, regularity of $S$ also implies that bidding $b_{1}\left(v_{1}, v_{2}\right)$ yields more expected surplus than abstaining from the first stage. The following proposition collects all of these results:

Proposition 8 (First-stage best-response characterization): The best response to any regular surplus function $S\left(v_{2}, c_{1}\right)$ is characterized by a bidding function $b_{1}\left(v_{1}, v_{2}\right) \geq L_{1}$ defined implicitly as $b_{1}\left(v_{1}, v_{2}\right)=v_{1}-S\left(v_{2}, b_{1}\left(v_{1}, v_{2}\right)\right)$.

The form of the bidding function in Proposition 8 provides interesting intuition. Since the firststage winner does not bid again, the option value $S$ of the second auction is the opportunity cost of winning the first item. Because of the truth-revealing property of the second-price auction, the bidder thus bids her net value of the first object, $v_{1}-S$, and the optimal bid does not depend on $G_{1}$. When evaluating the option value of the second auction, the bidder assumes she loses the first stage to a competitive bid that exactly matches her first bid. She thus assumes $c_{1}=b_{1}$ because $c_{1}=b_{1}$ is the only situation, in which changing the first bid slightly changes the outcome of the game, and $S\left(v_{2}, b_{1}\right)$ is thus the opportunity cost relevant at the margin.

The intuition why abstaining from the first auction is not a profitable deviation for any bidder follows from the regularity of $S$ : abstaining instead of bidding some $B \equiv b_{1}\left(v_{1}, v_{2}\right)>L_{1}$ changes the payoff only when the bidder would have won with $B$. Otherwise, the abstaining bidder would have advanced to the second stage anyway and the winner of the first auction would be the same as without the deviation, as well. Therefore, abstaining changes the outcome 
of the game for the focal bidder only when $c_{1}<B$. Then, the focal bidder gets $S\left(v_{2}, c_{1}\right)$ instead of $v_{1}-c_{1}$. But for all such low $c_{1}, v_{1}-S\left(v_{2}, c_{1}\right)>c_{1}$, so the deviation cannot be profitable (see Figure A2 in the Appendix for an illustration). For $c_{1}=L_{1}, v_{1}-S\left(v_{2}, c_{1}\right)=v_{1}>c_{1}$ (part $\mathrm{C}$ of regularity, Proposition 7). From continuity of $S$ in $c_{1}$ (part B of regularity), $v_{1}-S\left(v_{2}, c_{1}\right)>c_{1}$ for a range of small $c_{1}$ 's. And from the fact that there is always a unique solution to the equation (8), the inequality holds precisely for all $c_{1}<B$. In other words, deviation to abstaining changes the outcome of the game precisely when the highest competing bid is so low that losing the first auction results in a lower continuation surplus than winning it. Note that this is true even for bidder $\left(L_{1}+\varepsilon, H_{2}\right)$, who will win the second auction almost surely, and can only make $\varepsilon$ surplus in the first auction. Bidding $b_{1}\left(L_{1}+\varepsilon, H_{2}\right)>L_{1}$ makes sense even for this bidder, because she either loses anyway, or she wins when all other competitors bid even less than $b_{1}\left(L_{1}+\varepsilon, H_{2}\right)$. All competitors bidding that low implies that their $v_{2}$ s are so close to $H_{2}$ that winning the first auction is a better deal than competing with them.

Given Propositions 1-8, stating the main theorem of this paper is finally possible:

Theorem 1: There is a unique strictly monotone symmetric pure-strategy Bayesian Nash equilibrium with a continuous bidding function $b_{1}\left(v_{1}, v_{2}\right)$ that satisfies:

- $b_{1}\left(L_{1}, v_{2}\right)=L_{1}, b_{1}\left(v_{1}, L_{2}\right)=v_{1}$ and $v_{1}>b_{1}\left(v_{1}, v_{2}\right)>L_{1}$ for all $\left(v_{1}, v_{2}\right)>\left(L_{1}, L_{2}\right)$,

- $b_{1}\left(v_{1}, v_{2}\right)$ is decreasing in $v_{2}$ for all $\left(v_{1}, v_{2}\right)>\left(L_{1}, L_{2}\right)$, and

- $b_{1}\left(v_{1}, v_{2}\right)$ converges to $v_{1}$ as the number of bidders approaches infinity.

The proof of Theorem 1 essentially ties together all previous results, whereby the candidates for isobids analyzed above are elevated to the actual equilibrium isobids, and so is the candidate expected surplus function. The properties of a regular $S$ together with Proposition 4 imply all the properties of $b_{1}\left(v_{1}, v_{2}\right)$, where the comparative statics in valuations follow most easily by implicit differentiation of equation (8). The intuition for the comparative static in the number of bidders is as follows: The overall number of bidders $N$ controls the extent of the bid-shading in that as the number of bidders increases, winning the second auction becomes more difficult. 
Meanwhile, the winner's surplus also shrinks. Therefore, the expected surplus from the second auction shrinks to zero and the two auctions become effectively isolated.

The key to Theorem 1 is Proposition 3 that shows existence and uniqueness of candidate equilibrium isobids. The proof of Proposition 3 in turn relies on showing that a $K$-times repetition of the mapping $\boldsymbol{T}$ defined in equation (2) is a contraction map, so there is a natural iterative numerical method for computing isobids:

\section{Corollary to Theorem 1 (numerical procedure for computing $b_{1}\left(v_{1}, v_{2}\right)$ ):}

The following steps can be used to numerically approximate $b_{1}\left(v_{1}, v_{2}\right)$ on a grid:

1) Starting with $c_{1}=H_{1}$ and proceeding in small steps of size $\delta$, compute the equilibrium $I\left(v_{2} \mid c_{1}\right)$ for a set of $c_{1} \in\left\{L_{1}, L_{1}+\delta, L_{1}+2 \delta, \ldots, H_{1}\right\}$ by iterations of $\boldsymbol{T}\left(\cdot \mid c_{1}\right)$ starting with $\boldsymbol{T}\left(\cdot \mid c_{1}+\delta\right)$.

2) Construct equilibrium $S\left(v_{2}, c_{1}\right)$ by subtracting $c_{1}$ from $I\left(v_{2} \mid c_{1}\right)$.

3) Solve for $b_{1}\left(v_{1}, v_{2}\right)$ approximately on a grid using equation (8).

The first step makes use of continuity of $I$ in $c_{1}$ to initialize each set of iterations. Starting with $c_{1}=H_{1}$ is efficient because isobids for high values of $c_{1}$ have greater mass of $f$ underneath, and so their $\boldsymbol{T}\left(\cdot \mid c_{1}\right)$ contracts faster (see proof of Proposition 3). Budish (2008) uses this numerical procedure to assess the allocative efficiency of the sequential auction with information about future goods. He finds that the sequential auction is surprisingly efficient, realizing more than 98 percent of the potential gains from trade for a wide range of distributions with $v_{1}$ and $v_{2}$ uncorrelated.

\section{Example: Three bidders, $\boldsymbol{f}$ uniform on $[0,1] \times[0, K]$}

When $f$ is a uniform distribution on a rectangle and there are only three bidders, the equilibrium condition (2') yields an analytical solution for the equilibrium isobids because the condition implies a tractable differential-integral equation. The uniform distribution simplifies the integral 
under an isobid to: $\int_{0}^{I\left(v_{2} \mid c_{1}\right)} f\left(v_{1}, v_{2}\right) d v_{1}=\frac{\min \left[I\left(v_{2} \mid c_{1}\right), 1\right]}{K}$. Three bidders simplify the equilibrium condition because the crucial exponent is $N-2=1 .{ }^{9}$

In the Appendix, I show that these simplifications imply that the equilibrium isobids are $I\left(v_{2} \mid c_{1}\right)=c_{1} \cosh \left[A\left(c_{1}\right) v_{2}\right]$, where the function $A\left(c_{1}\right)$ satisfies $1=c_{1} A\left(c_{1}\right) \sinh \left(K A\left(c_{1}\right)\right)$ for $c_{1}$ low-enough that $I\left(K \mid c_{1}\right)<1$, and satisfies $K A\left(c_{1}\right)-\frac{1}{A\left(c_{1}\right)}=\log \left(1+\sqrt{1-c_{1}^{2}}\right)-\log \left(c_{1}\right)-\sqrt{1-c_{1}^{2}}$ for higher $c_{1}$. The two cases are necessary to capture the fact that isobids of low bid levels do not reach the upper bound of $v_{1}$ even for the highest possible $v_{2}$. With $K=1$, the cutoff is $c_{1}=0.552$, and it is easy to show that increasing $K$ reduces the cutoff.

Figure 2: Bidding function in the Uniform $[0,1] \times[0, K]$ example

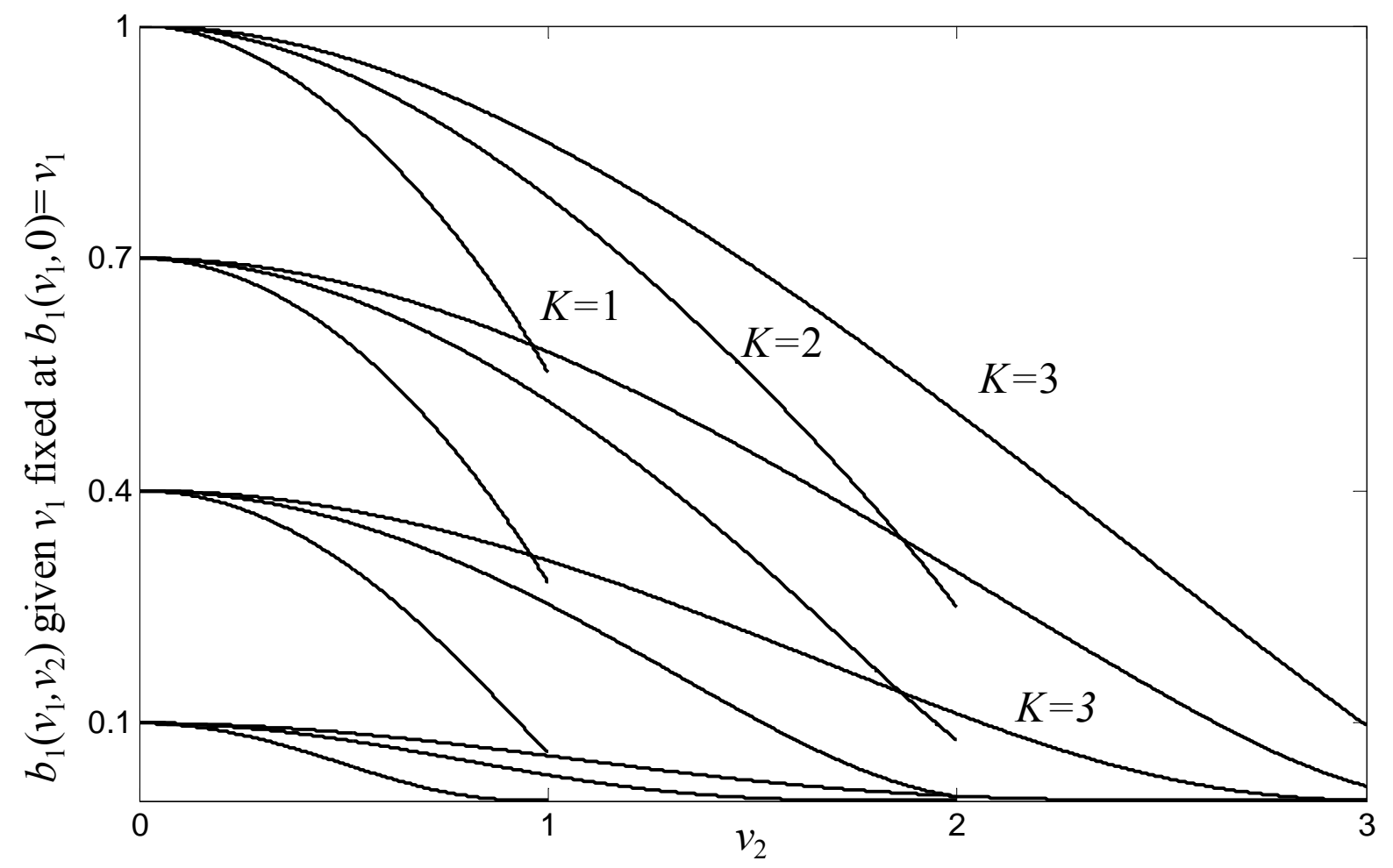

Note to Figure: Each solid line shows how $b_{1}\left(v_{1}, v_{2}\right)$ decreases as $v_{2}$ increases and $v_{1}$ remains fixed at $v_{1}=b_{1}\left(v_{1}, 0\right)$ for four levels of $v_{1}$ and three values of $K$. The $v_{1}=0.1$ bids asymptote towards zero as $v_{2}$ approaches $K$.

\footnotetext{
${ }^{9}$ The differential-equation approach for solving for the isobids analytically is complicated for $N>3$. Extending the approach used in the $N=3$ example would require solving a differential equation of the $(N-1)$ st order.
} 
Figure 2 shows representative bidding functions for $K=\{1,2,3)$ at four levels of $\mathrm{v}_{1}(0.1,0.40 .7$, and 1). For a given $\left(v_{1}, v_{2}\right)$, higher $K$ implies a higher equilibrium bid because there are more remaining competitors with higher $v_{2} \mathrm{~S}$ and hence less surplus. However, it is also clear from Figure 2 that higher $K$ involves higher expected surplus for the $\left(v_{1}, K\right)$ bidder, so the secondround competition is actually relatively weaker with higher $K$. It can be shown that the relative ordering is true for all $v_{2}$ s, namely that $b_{1}\left(v_{1}, v_{2} / K\right)$ decreases in $K$. However, note that every $\left(v_{1}, K\right)$ bidder still submits a positive first-round bid.

The Uniform example shows that a rectangular support $\left[L_{1}, H_{1}\right] \times\left[L_{2}, H_{2}\right]$ is more general than a square support $[L, H]^{2}$. Specifically, it is not sufficient to merely solve for $b_{1}\left(v_{1}, v_{2}\right)$ on a unit square (after appropriately rescaling the density $f$ to the unit square), and scale up the solution to an arbitrary rectangle $\left[L_{1}, H_{1}\right] \times\left[L_{2}, H_{2}\right]$. As the next proposition demonstrates, such a scaling procedure is available when both $v_{1}$ and $v_{2}$ are subjected to the same affine transform:

Proposition 9: Suppose $b_{1}\left(v_{1}, v_{2}\right)$ is the equilibrium bidding function for $f\left(v_{1}, v_{2}\right)$ with support on $\left[L_{1}, H_{1}\right] \times\left[L_{2}, H_{2}\right]$. Let $w_{i}=A+B v_{i}$ for some $A, B \geq 0$, and let $f_{w}\left(w_{1}, w_{2}\right)$ be the distribution of $\left(w_{1}, w_{2}\right)$. Then, $\tilde{b}\left(w_{1}, w_{2}\right)=A+B b_{1}\left(\frac{w_{1}-A}{B}, \frac{w_{2}-A}{B}\right)$ is the equilibrium bidding function for $f_{w}\left(w_{1}, w_{2}\right)$ on $\left[A+B L_{1}, A+B H_{1}\right] \times\left[A+B L_{2}, A+B H_{2}\right]$.

The key to preservation of the equilibrium bidding function under an affine transform is that the affine transform scales up both the intercept and the slope of the isobid by the same factor $B$. Thus, the affine transform of the equilibrium isobids in the $\left(v_{1}, v_{2}\right)$ space produces equilibrium isobids in the $\left(w_{1}, w_{2}\right)$ space: $I_{w}\left(w_{2} \mid c_{1}\right)=A+B I\left(\frac{w_{2}-A}{B} \mid \frac{c_{1}-A}{B}\right)$. Note that such preservation would not work if one affine transform were used on $v_{1}$ and another on $v_{2}$. For example, if $w_{1}=A+B v_{1} \quad$ and $\quad w_{2}=C+D v_{2}, \quad I_{w}\left(w_{2} \mid c_{1}\right) \neq A+B I\left(\frac{w_{2}-C}{D} \mid \frac{c_{1}-A}{B}\right) . \quad$ Therefore, proving equilibrium existence and uniqueness for a rectangular support is not a trivial extension of proving equilibrium existence for the unit square support. 
Besides the comparative statics in Theorem 1 and Proposition 9, I also see potential for a comparative static of $S\left(v_{2}, c_{1}\right)$ in the correlation between $v_{2}$ and $v_{1}$. Suppose the distribution $f$ is symmetric and its support is a square $[L, H]^{2}$. Consider the "exogenous" competition arising from $N-2$ bids independently drawn from the marginal population distribution of $v_{\mathrm{i}}$ : $F(v) \equiv F(H, v)=F(v, H)$. Exogenous competition is the competition anticipated by a forwardlooking bidder who does not appreciate the fact that future competitors are systematically selected in sequential auctions with information about future goods (and hence suffers from the loser's curse discussed in the Introduction). When $v_{2}$ and $v_{1}$ are independent, the equilibrium second-stage competition is stronger than exogenous because it involves the same number of bidders drawn from a distribution that first-order stochastically dominates $F$. The dominance follows from the fact that isobids are nondecreasing: for every $c_{1}$, independence implies that $F(v)=\frac{F\left(c_{1}, v\right)}{F\left(c_{1}, H\right)}$ and equation (4) defines the equilibrium distribution as

$$
\frac{F\left[I\left(\cdot \mid c_{1}\right), v\right]}{F\left[I\left(\cdot \mid c_{1}\right), H\right]}=\frac{F\left(c_{1}, v\right)+A}{F\left(c_{1}, H\right)+A+B}<\frac{F\left(c_{1}, v\right)}{F\left(c_{1}, H\right)}=F(v),
$$

where $A$ and $B$ are the positive probability-masses between $I\left(\cdot \mid c_{1}\right)$ and $c_{1}$ below $v$ and above $v$ respectively. In contrast to the independent case, $\frac{F\left[I\left(\cdot \mid c_{1}\right), v\right]}{F\left[I\left(\cdot \mid c_{1}\right), H\right]}>F(v)$ when $v_{2}$ and $v_{1}$ are sufficiently positively correlated, and so the second-stage competition becomes weaker than exogenous. In the limit as the correlation between $v_{2}$ and $v_{1}$ approaches unity, the identical-unit model of Milgrom and Weber (2000) applies. In their model, the bidder with the highest valuation $v=v_{1}=v_{2}$ exits in the first stage, so only the $N-1$ lowest bidders remain - weaker bidders than exogenous. 


\section{Extensions of the modeling assumptions}

\section{Free disposal of any won unit}

The model of section II assumes the first-stage winner cannot dispose of the first unit if she wins the second unit. This assumption is realistic for situations in which the goods are binding contracts for specialized procurement of products or services that are difficult to subcontract: fulfilling the first contract then ties up the capacity of the first-contract winner (Jofre-Bonet and Pesendorfer 2003). How would the results change if the goods were easily disposable, for example, two different consumer digital cameras? Let all assumptions remain exactly as in Section II, but assume disposal of any won object is free and the utility of owning both objects is $\max \left(v_{1}, v_{2}\right)$. Then the winner of the first unit will sometimes want to bid in the second auction because the second unit may provide additional value. In particular, the first-stage winner will bid $b_{2, W}\left(v_{1}, v_{2}\right)=\max \left(v_{1}, v_{2}\right)-v_{1}$ in the second stage, which is positive when $v_{2}>v_{1}$. The firststage losers, of course, will continue bidding $b_{2, L}\left(v_{1}, v_{2}\right)=v_{2}$ as in the model with costly disposal. In this subsection, I show that free disposal does not automatically guarantee trade in the first stage. Instead, symmetric equilibrium arguments analogous to Proposition 2 are still necessary to show that trade occurs almost surely. The concepts of a regular surplus function and an isobid extend readily to the free-disposal setting and make the analysis tractable.

Given the chance to throw away the first object and bid again, simply bidding a tiny $\varepsilon>0$ in the first stage seems costless even when $H_{2} \approx v_{2}>v_{1} \approx L_{1}$ : winning the first auction merely reduces the bidder's bid in the second auction rather than preventing bidding altogether. Instead of foregoing the entire expected surplus $S\left(v_{2}\right)$ by winning the first object, the winner with free disposal only foregoes the opportunity cost of $S\left(v_{2}\right)-S\left(v_{2}-v_{1}\right)$, which should be less than $v_{1}-$ the benefit of winning the first auction with a tiny $\varepsilon>0$. Therefore, free disposal seems to guarantee trade almost surely in any symmetric pure-strategy Nash equilibrium. Unfortunately, this intuition is incomplete because winning versus losing also impacts the second-stage competition: the winner faces stiffer competition than the losers. Contingent expected-surplus functions are necessary to characterize the equilibrium, as I discuss next.

First, define expected second-stage surpluses as integrals of the different winning probabilities in the cases of losing and winning the first auction (integrating by parts as in 
equation 4): Let $S_{L}\left(v_{2}, c_{1}\right)=\int_{0}^{v_{2}} G_{L}\left(c_{2} \mid c_{1}\right) d c_{2}$ be the expected second-stage surplus of the firststage loser, where $G_{L}$ is the cdf of $c_{2}$ in the case of losing the first stage. Analogously, let $S_{W}\left(v_{2}-v_{1}, c_{1}\right)=\int_{0}^{v_{2}-v_{1}} G_{W}\left(c_{2} \mid c_{1}\right) d c_{2}$ be the expected surplus of the first-stage winner with $G_{W}$. Since the winner faces stiffer second-stage competition than a loser (both face $N-2$ losers and one additional competitor, where the additional competitor is also a loser in $G_{W}$, as opposed to a winner in $G_{L}$, and winners bid less than losers), $G_{W}$ stochastically dominates $G_{L}: G_{L} \geq G_{W}$. Given any $S_{L}$ and $S_{W}$, the bidders solve the following problem in the first stage:

$$
b_{1}\left(v_{1}, v_{2}\right)=\underset{\beta}{\arg \max }\left\{\int_{0}^{\beta}\left[v_{1}-c_{1}+\mathbf{1}\left(v_{2}>v_{1}\right) S_{W}\left(v_{2}-v_{1}, c_{1}\right)\right] d G_{1}\left(c_{1}\right)+\int_{\beta}^{\infty} S_{L}\left(v_{2}, c_{1}\right) d G_{1}\left(c_{1}\right)\right\}
$$

Compared to equation (7), winning the first auction involves the additional surplus $S_{W}\left(v_{2}-v_{1}, c_{1}\right)$. The first-order conditions are only different from equation (8) with $S_{L}$ instead of $S$ when $v_{2}>v_{1}$ :

$$
\begin{aligned}
& F O C \mid v_{2} \leq v_{1}: \beta=v_{1}-S_{L}\left(v_{2}, \beta\right) \\
& F O C \mid v_{2}>v_{1}: \beta=\left\{v_{1}-\left[S_{L}\left(v_{2}, \beta\right)-S_{L}\left(v_{2}-v_{1}, \beta\right)\right]\right\}+\left[S_{W}\left(v_{2}-v_{1}, \beta\right)-S_{L}\left(v_{2}-v_{1}, \beta\right)\right]
\end{aligned}
$$

The incomplete intuition of the previous paragraph notes that the term in the curly brackets must be positive for any $S_{L}$ that is an integrated cdf. The intuition was incomplete because the second term (in the square brackets) is always negative due to the fact that $G_{L} \geq G_{W}$. The $F O C$ corrects for the increased competition faced by the winner of the first stage relative to the losers. This makes winning the first stage less attractive than only considering the situation of the losers, and there is no immediate guarantee that a solution with $\beta>0$ exists. Instead, I need an argument analogous to the one for Propositions 2 and 3 in order to guarantee first-stage trade almost surely, along with the appropriate regularity conditions on $S_{L}$ and $S_{W}$. I do not develop the existence and uniqueness proof in detail; it is analogous to that in Section II. The intuition is more important: the endogenous option value created by the information about future goods can 
make simple single-agent intuition about best response to exogenous competition unreliable. Instead of relying on such intuition for even qualitative predictions about market equilibria, it is necessary to analyze the link between first-stage bidding and the second-stage option value.

The isobids of the free-disposal model are weakly bounded below by the isobids of the costly disposal model. For all $c_{1}$ such that $I\left(v_{2} \mid c_{1}\right) \geq v_{2}$, the isobids of the two cases coincide exactly, and $S_{L}\left(v_{2}, c_{1}\right)=S\left(v_{2}, c_{1}\right)$ of Section II because the winner does not bid again when her $v_{1} \geq v_{2}$. When $c_{1}$ is low enough that $I\left(v_{2} \mid c_{1}\right)<v_{2}$ with costly disposal, $S_{L}\left(v_{2}, c_{1}\right)<S\left(v_{2}, c_{1}\right)$ because of the additional competition from the winner. The equilibrium condition becomes

$$
\tilde{I}^{\prime}\left(v_{2} \mid c_{1}\right)=G_{L}\left(v_{2} \mid c_{1}\right)-\mathbf{1}\left(I<v_{2}\right) G_{W}\left[v_{2}-\tilde{I}\left(v_{2} \mid c_{1}\right) \mid c_{1}\right]
$$

and it is clear that $I\left(v_{2} \mid c_{1}\right)$ of Section II does not coincide with $\tilde{I}\left(v_{2} \mid c_{1}\right)$, and $\tilde{I}\left(v_{2} \mid c_{1}\right)<I\left(v_{2} \mid c_{1}\right)$ because the isobids shade the same intercept, and $\tilde{I}$ has lower slope everywhere. Note that because $v_{1}$ appears in the limits of integration in (11), the equilibrium condition (12) is less elegant than that in (2). The reason is that the constraint $c_{1}$ imposes on the first-auction-winner's valuations is that her $\left(v_{1}, v_{2}\right)$ lie on the $c_{1}$ isobid contour (as opposed to weakly underneath it as in the case of other losers). Because of the resulting restriction of a competitor to a measure-zero set in the $\left(v_{1}, v_{2}\right)$ space, the model likely breaks down for distributions with discontinuous densities, but the existence and uniqueness ideas of Section II should go through under the assumption of continuous $f$.

Even without solving the model further, it is obvious from $\tilde{I}\left(v_{2} \mid c_{1}\right) \leq I\left(v_{2} \mid c_{1}\right)$ that when disposal is free and the equilibrium exists, the equilibrium first-stage bid $b_{1}\left(v_{1}, v_{2}\right)$ is at least as high as the corresponding first-stage bid given the same $\left(v_{1}, v_{2}\right)$ in the model with costly disposal. At least in the $[f$ Uniform, $\mathrm{N}=3]$ example, the first-stage bidding strategies are surprisingly similar with and without free disposal, the biggest absolute differences predictably occurring in the $\left(v_{1}<v_{2}\right)$ region of the parameter space. See Figure 3 for an illustration. 
Figure 3: First-stage bidding function with $f=$ Uniform $[0,1]^{2}$ and 3 bidders.

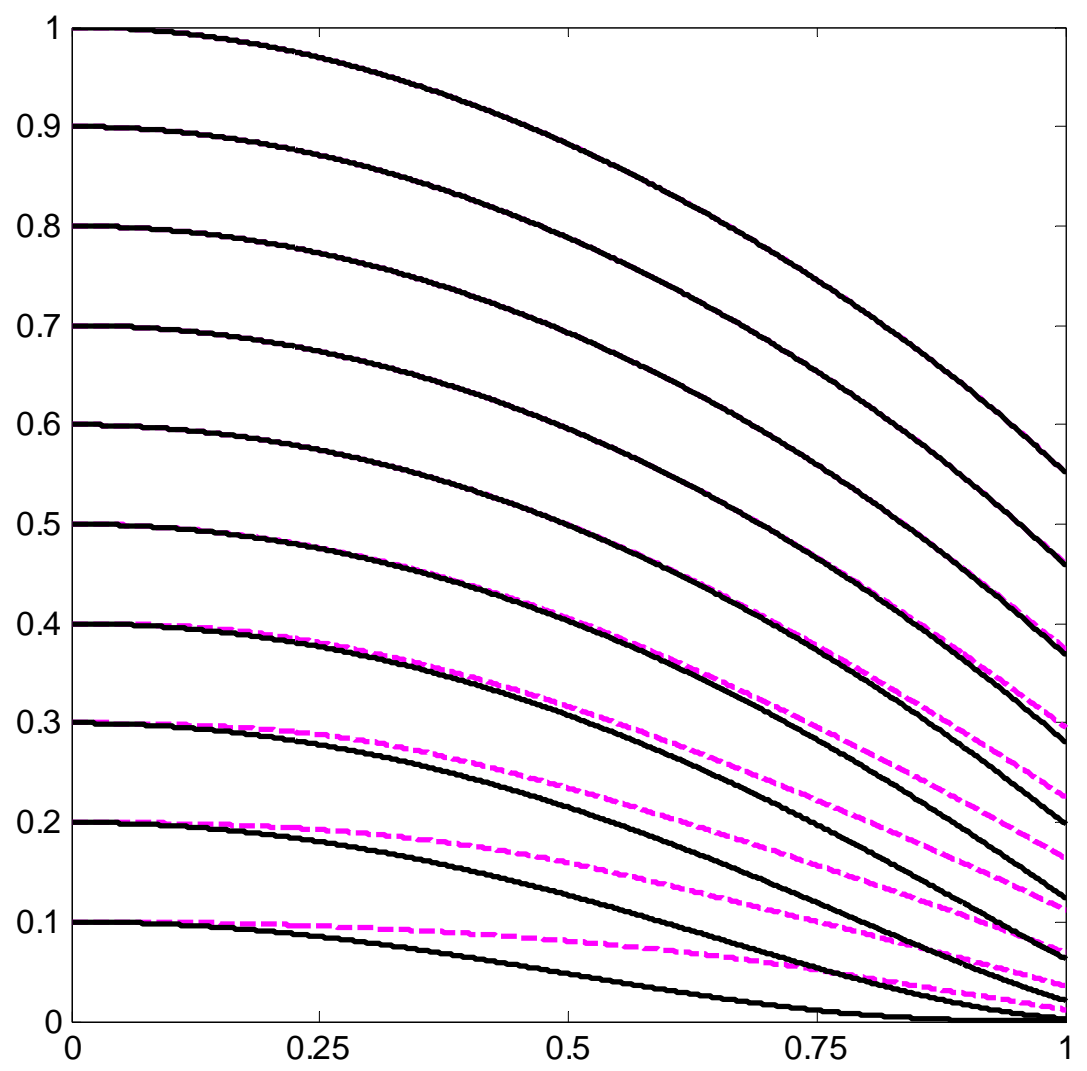

Note to Figure: Each solid line shows how $b_{1}\left(v_{1}, v_{2}\right)$ decreases as $v_{2}$ increases and $v_{1}$ remains fixed at $v_{1}=b_{1}\left(v_{1}, 0\right)$. The dashed (magenta) lines are defined analogously, and illustrate the freedisposal case.

\section{First-stage reserve prices above $L_{1}$}

Let all assumptions remain exactly as in Section II, but assume the seller uses a reserve-price $R>L_{1}$ in the first stage. Trade is no longer guaranteed because bidders with $v_{1}<R$ cannot derive a positive surplus from the first auction. More surprisingly, the reserve also changes the bidding strategy of those bidders who participate in that a positive measure of the bidders pool on bidding exactly $R$.

For bid-levels above $R$, the isobids of Section II capture bidding even with the reserve because losing the auction at the margin still implies encountering $N-2$ competitors from below 
the isobid in the second stage. Unlike without the reserve, bidders along $I\left(v_{2} \mid R\right)$ are not the only ones who bid $R$ : any bidder just below $I\left(v_{2} \mid R\right)$ would rather bid $R$ than abstain, because he either loses anyway or avoids the strong competition should all $\mathrm{N}-1$ competitors abstain as well. Therefore, there must be a positive area of the valuation space, in which bidders bid exactly $R$, and $I\left(v_{2} \mid R\right)$ is the upper boundary of this area. Denote the lower boundary of this area $I_{A}\left(v_{2}\right)$. To complete the equilibrium construction, $I_{A}\left(v_{2}\right)$ must be the indifference line between abstaining and bidding $R$. Suppose such a line exists, and let $N$-1 competitors use the line in guiding their participation decisions. Consider a bidder, who best responds to such behavior. If she abstains, she encounters either $N-1$ other abstainers in the second stage or $N-2$ exogenous competitors (in case at least one competitor does not abstain, see the end of Section II for a definition of an exogenous competitor). The resulting expected surplus is $\alpha \int_{L_{2}}^{v_{2}}\left(\frac{F\left(I_{A}, x\right)}{F\left(I_{A}, H_{2}\right)}\right)^{N-1} d x+(1-\alpha) \int_{L_{2}}^{v_{2}} F^{N-2}\left(H_{1}, x\right) d x$ where $\alpha=F^{N-1}\left(I_{A}, H_{2}\right)$ is the probability that all $N$-1 bidders abstain, and $\frac{F\left(I_{A}, x\right)}{F\left(I_{A}, H_{2}\right)}$ is the distribution of the $v_{2}$ of each competitor who abstains (by equation 3). If the bidder bids $R$ instead of abstaining, then either nothing changes if she loses the first auction anyway (if $c_{1}>R$ or if $c_{1}=R$ and the highest competitor wins the first auction in a tie), or she wins the first object and receives surplus $v_{1}-R$ instead of $\int_{L_{2}}^{v_{2}}\left(\frac{F\left(I_{A}, x\right)}{F\left(I_{A}, H_{2}\right)}\right)^{N-1} d x$. Therefore, the indifference line $I_{A}\left(v_{2}\right)$ must satisfy a slightly different condition than equation (2):

$$
I_{A}\left(v_{2}\right)=\tilde{\boldsymbol{T}}^{K}(\cdot \mid R)=R+\int_{L_{2}}^{v_{2}}\left(\frac{F\left(I_{A}, x\right)}{F\left(I_{A}, H_{2}\right)}\right)^{N-1} d x
$$

The line $I_{A}\left(v_{2}\right)$ exists whenever $I\left(v_{2} \mid R\right)$ does because the contraction property of $\boldsymbol{T}^{K}(\cdot \mid R)$ of Proposition 3 for some $K$ implies that the mapping $\tilde{\boldsymbol{T}}^{K}$ defined in equation (13) is also a contraction. Moreover, it is clear that $R<I_{A}\left(v_{2}\right)<I\left(v_{2} \mid R\right)$, so there is a positive measure of bidders who bid exactly $R$. These results are summarized in the following proposition: 
Proposition 10: When the seller uses a reserve price above $L_{1}$ in the first stage, there is a unique symmetric pure-strategy Bayes Nash equilibrium $b_{1}^{R}\left(v_{1}, v_{2}\right)$ that satisfies:

- $b_{1}^{R}\left(v_{1}, v_{2}\right)=b_{1}\left(v_{1}, v_{2}\right)$ when $\left(v_{1}, v_{2}\right)$ above $I\left(v_{2} \mid R\right)$

- $b_{1}^{R}\left(v_{1}, v_{2}\right)=R$ when $\left(v_{1}, v_{2}\right)$ between $I\left(v_{2} \mid R\right)$ and $I_{A}\left(v_{2}\right)$

- abstain from the first auction when $\left(v_{1}, v_{2}\right)$ below $I_{A}\left(v_{2}\right)$

Several properties of the bidders' response to the reserve contrast with the standard results obtained with uni-dimensional types by Myerson (1981) for a single unit and Masking and Riley (1989) for multiple identical units. First, the reserve on the first object is not equivalent to screening on the valuation of the first object at the reserve. Instead, bidders are screened according to equation (13), which screens some bidders with $v_{1}>R$ as well. Second, despite the auction being second-price sealed-bid, the reserve changes the bidding strategy of bidders above the screening level: a positive measure of bidders pools on bidding $R$ exactly. This observation is surprising at first because the truth-revealing nature of a standard single-shot 2PSB suggests a reserve price should not affect bidding. However, the intuition behind the partial pooling is analogous to that in models of auctions with positive externalities (for example, Jehiel and Moldovanu 2000, Haile 2000), where partial pooling by certain low bidders also happens. In the case of a second auction as a positive externality, pooling occurs because the "low" types between $I\left(v_{2} \mid R\right)$ and $I_{A}\left(v_{2}\right)$ would rather win the first auction should everyone also abstain, but would rather abstain should someone bid at least $R$. As in the literature on auctions with externalities, the optimal reserve price will likely depend on the number of bidders.

Because of the multi-dimensional types, a simple characterization of the optimal reserve in a direct revelation mechanism as in Masking and Riley (1989) is not feasible. To investigate the optimal reserve at least numerically, I once again focus on the uniform case and simulate bidding for all possible reserve levels. Figure 4 illustrates Proposition 10 in the uniform case with 3 bidders and the reserve set at 0.5 - the profit-maximizing level in the absence of the second stage (Myerson 1981). It is clear from the figure that there is a substantial mass of bidders bidding exactly reserve, and the probability of screening is greater than half. Despite this amount of pooling and screening, I find that the reserve level optimal for the first-stage revenue is still approximately 0.5 regardless of the number of bidders. Since increasing the reserve increases 
abstentions and hence competition in the second stage, the second-stage revenue increases in the first-stage reserve. Therefore, the first-stage reserve optimal for total revenue is strictly above the level optimal for the marginal distribution of valuations. The simulations indicate that the optimal reserve is 0.55 for 3 bidders and 0.53 for 4 bidders. Further analysis of optimal reserves is beyond the scope of this paper, but would make for an important contribution in future work.

\section{Figure 4: Isobids with a first-stage reserve ( $f=\operatorname{Uniform}[0,1]^{2}, N=3$ bidders)}

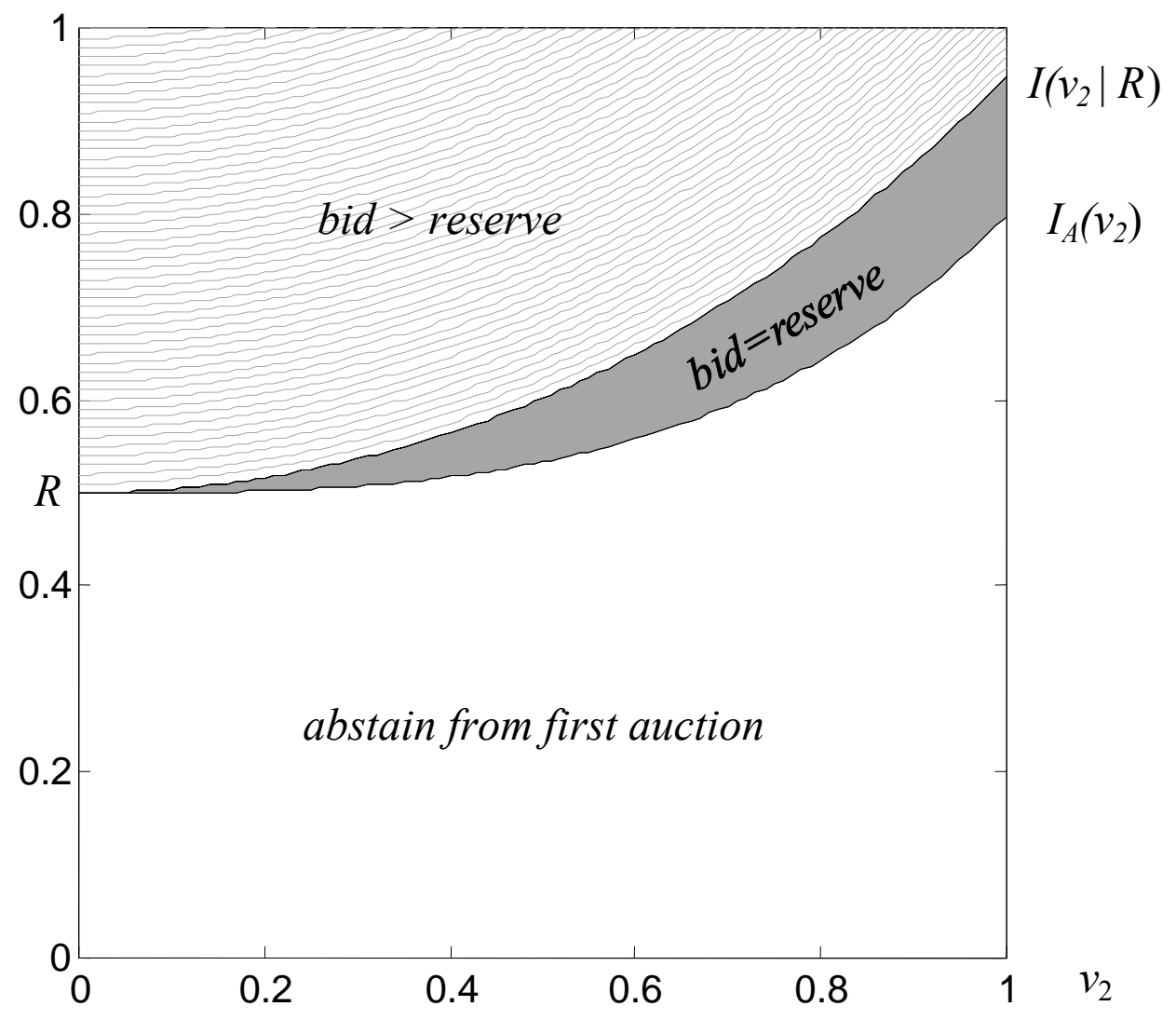

Note to Figure: The first-stage reserve is $R=0.5$. Bidders in the shaded area bid exactly reserve in the first stage. Bidders above $I\left(v_{2} \mid R\right)$ bid as they would without the reserve, the thin gray lines show their isobids at 0.01 intervals. Bidders below $I_{A}\left(v_{2}\right)$ abstain from the first auction.

\section{More than two auctions in the sequence}

The asymmetry and learning issues Milgrom and Weber (2000) first raised in their seminal paper complicate the analysis of more than two auctions in the current model as well. Suppose there are three auctions instead of only two, with each bidder having private single-item valuations $\left(v_{1}, v_{2}, v_{3}\right)$, and realistically suppose further that only the winner and the price $p_{1}$ of the 
first unit are revealed before the second stage. Then, second-stage bidders have asymmetric beliefs about the $v_{3}$ of the remaining competitors, because one of the remaining bidders bid exactly $p_{1}$ while the other bidders bid strictly below $p_{1}$. Even when this asymmetry is resolved by revealing all first-stage bids as suggested by Milgrom and Weber, the information about future goods would make it necessary to explicitly model second-stage beliefs about $v_{3}$ : First-stage bids would be a function of $v_{3}$, and bidders thus may have an incentive to mislead their competitors into thinking their $v_{3}$ is very high by bidding very low in the first stage. Even the three-auction series would thus be a strategically rich environment, the price $p_{1}$ would enter second-stage bids, and whether symmetric pure-strategy equilibria would exist in the first stage is unclear.

\section{Discussion}

As auction-driven marketplaces become more important in the selling of both procurement contracts by firms and governments and in the selling of consumer goods on the Internet, it is important to understand the implication of their information structure on bidding behavior, market efficiency, and distribution of the realized social surplus. This paper provides a model of forward-looking bidding in a sequence of two auctions when the characteristics of the good sold in the upcoming auction are announced in advance, allowing each bidder to form a private valuation of the upcoming good before bidding in the first auction. This information structure is common in the real world, and it has a profound impact on the nature of bidding. While it is complicated to properly account for the systematic selection of future competitors by first-stage bids, the overall result is surprisingly simple: for any distribution with a density and full support on a compact rectangle, there always exists a unique strictly monotone pure-strategy equilibrium with mostly intuitive properties.

Without any restrictions on the distribution of bidder types other than full support, the equilibrium bidding has the following intuitive properties: First, equilibrium bids increase in the value of the first object and decrease in the value of the second object. Specifically, bidders bid their private values of today's object minus the expected surplus of tomorrow's auction, where the expected surplus function increases convexly in the valuation of tomorrow's unit and, less intuitively, depends on the valuation of today's unit. The expected surplus function implicitly depends on the valuation of today's unit because it is optimal for each bidder to assume that she is pivotal to the outcome of the game (a natural assumption in a second-price sealed-bid auction), 
and hence should she lose today's auction, she would lose in a tie with today's maximum competitive bid. Other general properties of the equilibrium bidding function include stability under affine transformation of the valuations, and comparative statics in the number of bidders and the correlation of valuations: As the number of bidders increases, the surplus function vanishes, so the auctions become effectively isolated. Negative correlation between the two private valuations also increases first stage bids, for a similar reason: the stronger the correlation, the stronger the equilibrium competition in the second stage, resulting in less expected surplus to losers of the first stage, and hence smaller opportunity cost of winning.

The main counter-intuitive property of the equilibrium is that trade happens almost surely in the first auction. Bidders do not abstain from the first auction because of the loser's curse arising from the informational link between today's bid-level and future competition. In the equilibrium, losing to very small bids implies a second-round competition so strong that winning the first object is a better deal. To correct for the loser's curse that would arise from ignoring this "bad news" about the continuation payoff, it is optimal for bidders to bid more aggressively. Not only is optimal to bid a positive amount in the first auction in equilibrium, it only makes sense to bid more than the lower bound of the support of the first-object valuations $\left(L_{1}\right)$. This is because in a symmetric pure-strategy equilibrium, any bid-level $\beta$ between zero and $L_{1}$ would imply the existence of a bidder who is guaranteed to encounter only strictly higher $v_{2}$ 's than his own should he lose while bidding $\beta$, and so her expected second-stage surplus is zero on the margin. The best response to such a strong loser's curse is to bid the valuation of the first object in the first stage, which must be at least $L_{1}$.

The main model (Section II) assumes that the first-stage winner cannot dispose of the won object and bid again in the second stage. Such a costly assumption is most realistic for situations in which the goods are binding contracts for specialized procurement of products or services that are difficult to subcontract. It may seem that allowing free disposal of any unit would ensure trade because any bidder could bid a very small $\varepsilon>0$ in the first stage, participate in the second stage, and throw away the lesser-valued object should she win both. The extension of the model for free disposal (Section IV) demonstrates that this reasoning is incomplete because of the systematic selection of second-round competitors: the first-auction winner always faces a tougher competition in the second round than a first-auction loser. Despite this 
complication, the arguments developed in Section II can be extended to show that there exists a unique strictly monotone pure-strategy equilibrium even in the case of free disposal.

A fruitful area of future work is the question of optimal auctioning to the multidimensional bidders in the present model. Jehiel et al (1999) demonstrate that the general optimal incentive-compatible design can be very complex. A second best approach to optimal selling may be to at least optimize the first-stage reserve price. I show that the modeling approach based on isobids generalizes readily to a first-stage reserve inside the support of the first-object valuations, as does the computational technique. Using the generalized technique, I explore the possible reserve-levels, and find that, at least in the uniform case, the optimal firststage reserve depends on the number of bidders and exceeds the level optimal for sale of a single object to bidders drawn from the marginal distribution.

Generalizing the model to more than two stages under the current assumptions would be cumbersome. When there are more than two auctions in a sequence, the bidders still shade their bids in expectation of a positive future surplus. However, the optimal bidding strategies are no longer symmetric and no longer depend only on each bidder's private information, because there is useful information in prices and other experiences bidders encounter along the way. However, at least a limited extension of the proposed model to many stages would be desirable. 


\section{Appendix: Proofs of propositions}

Proof of Proposition 2: Suppose such a candidate isobid $I$ exists. Note that equation (2) implies that any candidate isobid is an integrated cumulative density function, and so it must be continuous and nondecreasing. Equation (2) implies that $I\left(L_{2} \mid \beta\right)=\beta<L_{1}$. Since $I\left(v_{2} \mid c_{1}\right)>L_{1}$ for some $v_{2} \in\left(L_{2}, H_{2}\right]$, continuity of $I$ implies that there is a $v^{*}<v_{2}$ such that $I\left(v^{*} \mid \beta\right)=L_{1}$. Since $I$ is nondecreasing, $F[I, w]=0$ for every $w \leq v^{*}$. In other words, all bidders along $I$ with $v_{2} \leq v^{*}$ are guaranteed to encounter strictly superior competitors in the second round with probability one (should they lose on the margin at bid-level $\beta$ ). Therefore, the RHS of equation (2) is $\beta$ for all $v_{2} \leq v^{*}$, which is a contradiction with $I\left(v^{*} \mid \beta\right)=L_{1}$. QED Proposition 2.

Proof of Proposition 3: Fix any $c_{1} \in\left(L_{1}, H_{1}\right)$ and denote the global bound on $f$ by $\bar{f}$. Let $B\left(c_{1}\right)$ be a set of nondecreasing 1-Lipschitz functions $I:\left[L_{2}, H_{2}\right] \rightarrow\left[c_{1}, c_{1}+H_{2}-L_{2}\right]$ such that $I\left(L_{2}\right)=c_{1}: B\left(c_{1}\right)=\left\{I:\left[L_{2}, H_{2}\right] \rightarrow\left[c_{1}, c_{1}+H_{2}-L_{2}\right], I\left(L_{2}\right)=c_{1}, \forall x>y, 0 \leq I(x)-I(y)<x-y,\right\}$

$B\left(c_{1}\right)$ is a closed subset of the complete metric space of all bounded continuous functions from $\left[L_{2}, H_{2}\right]$ to $\left[0, H_{1}+H_{2}-L_{2}\right]$ with the supremum metric: $d(I, J)=\max _{v \in\left[L_{2}, H_{2}\right]}[|I(v)-J(v)|]$, so it is a complete metric space with the same metric. It is immediate from equation 2 that $\boldsymbol{T}$ projects $B\left(c_{1}\right)$ into itself: $\boldsymbol{T}: B\left(c_{1}\right) \rightarrow B\left(c_{1}\right)$. The remainder of the proof shows that for every $c_{1} \in\left(L_{1}, H_{1}\right)$, a $K \geq 1$ exists such that $\boldsymbol{T}^{K}(I)$ is a contraction map. That is, a $q<1$ exists such that $d\left(\boldsymbol{T}^{K}(I), \boldsymbol{T}^{K}(J)\right)<q d(I, J)$ for all $I, J \in B\left(c_{1}\right)$. By the Banach Fixed Point Theorem, this is enough to show that $\boldsymbol{T}$ has a unique fixed point in $B\left(c_{1}\right)$ and iterations of $\boldsymbol{T}$ starting at any point in $B\left(c_{1}\right)$ converge to the unique fixed point exponentially fast. The unique fixed point is the unique isobid $I\left(v_{2} \mid c_{1}\right)$.

It is enough to consider $N=3, d(\boldsymbol{T}(I), \boldsymbol{T}(J))$ obviously decreases in $N$. Pick any distance $\delta>0$ and any $I_{1} \in B\left(c_{1}\right)$. Let $I_{2}(v)=\underset{J \in B\left(c_{1}\right)}{\arg \max } d\left(\boldsymbol{T}^{K}\left(I_{1}\right), \boldsymbol{T}^{K}(J)\right)=I_{1}(v)+\min [\delta, v]$, 
and note that $d\left(\boldsymbol{T}^{K}\left(I_{1}\right), \boldsymbol{T}^{K}\left(I_{2}\right)\right)<d\left(\boldsymbol{T}^{K}\left(I_{1}\right), \boldsymbol{T}^{K}\left(I_{1}+\delta\right)\right)$. Therefore, it is sufficient to show $d\left(\boldsymbol{T}^{K}(I), \boldsymbol{T}^{K}(I+\delta)\right)<q \delta$ for some $q<1$. The following Lemma is critical for the result:

Lemma $1: \forall \alpha \in R_{+} \&$ integer $M \geq 0:\left|\boldsymbol{T}\left(I(x)+\frac{\alpha x^{M}}{M !}\right)(w)-\boldsymbol{T}(I(x))(w)\right|<\frac{\alpha \bar{f}^{(M+2)}}{(M+2) ! F\left(c_{1}, 1\right)}$

Proof: $\left|\boldsymbol{T}\left(I(x)+\frac{\alpha x^{M}}{M !}\right)(w)-\boldsymbol{T}(I)(w)\right|=\int_{L_{2}}^{w}\left[\frac{\int_{L_{2}}^{z}\left[\int_{L_{2}}^{I\left(v_{2}\right)+\frac{\alpha v_{2}^{M}}{M !}} f\left(v_{1}, v_{2}\right) d v_{1} d v_{2}\right.}{\int_{L_{2}}^{I\left(v_{2}\right)+\frac{\alpha v_{2}^{M}}{M !}} \int_{L_{1}}^{M !} f\left(v_{1}, v_{2}\right) d v_{1} d v_{2}} \int_{L_{2}}^{I\left(v_{2}\right)} f\left(v_{L_{1}}^{H_{2}} \int_{L_{1}}^{I\left(v_{2}\right)} f\left(v_{1}\right) d v_{1} d v_{2}\right) d v_{1} d v_{2}\right] d z$

$<\frac{\int_{\left(I>c_{1}\right)}^{w} \int_{L_{2}}^{w} \int_{L_{2}}^{I\left(v_{2}\right)+\frac{\alpha v_{2}^{M}}{M !}} \int_{I\left(v_{2}\right)}^{M !} f\left(v_{1}, v_{2}\right) d v_{1} d v_{2} d z}{F\left(c_{1}, 1\right)}<\frac{\int_{(f<\bar{f})}^{w} \int_{L_{2}}^{w} \int_{L_{2}}^{z} \alpha \bar{f} \frac{v_{2}^{M}}{M !} d v_{2} d z}{F\left(c_{1}, 1\right)}<\frac{\alpha \bar{f} \int_{0}^{w} \int_{0}^{z} v_{2}^{M} d v_{2} d z}{M ! F\left(c_{1}, 1\right)}=\frac{\alpha \bar{f} w^{(M+2)}}{(M+2) ! F\left(c_{1}, 1\right)}$

Lemma 1 implies $\boldsymbol{T}$ may not be a contraction for small-enough $c_{1}$ : setting $\alpha=\delta$ and $M=0$ in

Lemma 1 implies $d(\boldsymbol{T}(I), \boldsymbol{T}(I+\delta))=\left|\boldsymbol{T}(I)\left(H_{2}\right)-\boldsymbol{T}(I+\delta)\left(H_{2}\right)\right|<\frac{\delta \bar{f} H_{2}^{2}}{2 F\left(c_{1}, 1\right)}$, and $\frac{\delta \bar{f} H_{2}^{2}}{2 F\left(c_{1}, 1\right)}$

may not be less than unity. However, note that $\boldsymbol{T}$ bounds the difference between the images of $I$ and $I+\delta$ quadratically as a function of $v:|\boldsymbol{T}(I)(v)-\boldsymbol{T}(I+\delta)(v)|<\frac{\delta \bar{f}^{2}}{2 F\left(c_{1}, 1\right)}$. The quadratic bound strengthens to quartic when one applies $\boldsymbol{T}$ twice, that is, when $\boldsymbol{T}$ is applied to $\boldsymbol{T}(I+\delta)$ and $\boldsymbol{T}(I)$ :

$\left|\boldsymbol{T}^{2}(I+\delta)(w)-\boldsymbol{T}^{2}(I)(w)\right|<\left|\boldsymbol{T}\left(J(v)+\frac{\delta \bar{f} v^{2}}{2 F\left(c_{1}, 1\right)}\right)(w)-\boldsymbol{T}(J(v))(w)\right|<\delta \frac{w^{4}}{4 !}\left(\frac{\bar{f} H_{2}^{2}}{F\left(c_{1}, 1\right)}\right)^{2}$,

where $\quad J=\boldsymbol{T}(I) . \quad$ By induction, $\quad d\left(\boldsymbol{T}^{K}(I), \boldsymbol{T}^{K}(I+\delta)\right)<\frac{\delta}{(2 K) !}\left(\frac{\bar{f} H_{2}^{2}}{F\left(c_{1}, 1\right)}\right)^{K} . \quad$ Since $\lim _{K \rightarrow \infty} \frac{C^{K}}{(2 K) !}=0$ for every positive constant $C$, this limit as $K$ grows proves a $K$ exists high enough that $\boldsymbol{T}^{K}$ is a contraction. QED Proposition 3. 
Proof of Proposition 5: Suppose there is a pair $d>c$ and a $\tilde{v} \in\left[L_{2}, H_{2}\right]$ such that $I(v \mid d) \leq I(v \mid c)$, and denote $I_{c}(v) \equiv I(v \mid c)$. Continuity of isobids implies that the two isobids must intersect at or below $\tilde{v}$. Let the smallest intersection point be $v^{*}$, namely, $I_{d}(v)>I_{c}(v)$ for all $v<v^{*}$ and $I_{d}\left(v^{*}\right)=I_{c}\left(v^{*}\right)$. Therefore, $I_{c}(v)$ intersects $I_{d}(v)$ from below at $v^{*}$, and so the slope of $I_{c}(v)$ at $v^{*}$ must be weakly higher than the slope of $I_{d}(v)$ at $v^{*}: I_{c}^{\prime}\left(v^{*}\right) \geq I_{d}^{\prime}\left(v^{*}\right)$. Equilibrium and full support of $f$ rule this ordering of slopes at $v^{*}$ out. Three distinct cases exist: Case 1: $I_{d}(v) \leq I_{c}(v)$ on $\left(v^{*}, H_{2}\right]$ (see dotted line in Figure A2 accompanying this proof). From equation $6, I_{b}^{\prime}\left(v^{*}\right)=G_{2}\left(v^{*} \mid I_{b}\right)=\left(\frac{F\left(I_{b}, v^{*}\right)}{F\left(I_{b}, H_{2}\right)}\right)^{N-2}$. Let $A=F\left(\min \left[I_{c}, I_{d}\right], H_{2}\right), A 1=F\left(I_{c}, v^{*}\right)$, and $X=F\left(I_{d}, v^{*}\right)-F\left(I_{c}, v^{*}\right)$. From full support of $f, X>0$. Since $I_{d}(v) \leq I_{c}(v)$ on $\left(v^{*}, H_{2}\right]$, $I_{d}^{\prime}\left(v^{*}\right)=\left(\frac{A 1+X}{A+X}\right)^{N-2}>\left(\frac{A 1}{A}\right)^{N-2} \geq\left(\frac{A 1}{A+B+Y}\right)^{N-2}=I_{c}^{\prime}\left(v^{*}\right)$, where $B+Y \geq 0$ is the probability mass between the two isobids on $\left(v^{*}, H_{2}\right]$, and it is non-negative because of full support and $I_{d}(v) \leq I_{c}(v)$ on $\left(v^{*}, H_{2}\right]: B+Y=\left[F\left(I_{c}, H_{2}\right)-F\left(I_{c}, v^{*}\right)\right]-\left[F\left(I_{d}, H_{2}\right)-F\left(I_{d}, v^{*}\right)\right]$. This inequality based on the equilibrium relation is a contradiction with the ordering of slopes necessary for an intersection at $v^{*}$. Intuitively, a single intersection at $v^{*}$ forces the conditional probability of a $v_{2}<v^{*}$ greater for the higher and flatter isobid, and this conditional probability happens to be the slope of the isobid at $v^{*}$.

Case 2: Suppose the ordering of the isobids on $\left(v^{*}, H_{2}\right]$ is ambiguous. At least one more intersection must be at point $w^{*}>v^{*}$, and it must be true that $I_{c}^{\prime}\left(w^{*}\right) \leq I_{d}^{\prime}\left(w^{*}\right)$. For equilibrium isobids, the order of slopes at $v^{*}$ and the full support rule out this ordering of slopes at $w^{*}$. Let $A 2+B 1=F\left(I_{d}, w^{*}\right)-F\left(I_{d}, v^{*}\right), Y=\left[F\left(I_{c}, w^{*}\right)-F\left(I_{c}, v^{*}\right)\right]-\left[F\left(I_{d}, w^{*}\right)-F\left(I_{d}, v^{*}\right)\right]$, and $Q=\left[F\left(I_{d}, H_{2}\right)-F\left(I_{d}, w^{*}\right)\right]-\left[F\left(I_{c}, H_{2}\right)-F\left(I_{c}, w^{*}\right)\right]$ (see Figure A2). From full support of $f$, $A 2+B 1>0$ and $Y>0$, but the sign of $Q$ is ambiguous. Nevertheless, we can express $I_{c}^{\prime}\left(v^{*}\right) \geq I_{d}^{\prime}\left(v^{*}\right)$ as $I_{c}^{\prime}\left(v^{*}\right)=\left(\frac{A 1}{A+B+Y}\right)^{N-2}>\left(\frac{A 1+X}{A+B+X+Q}\right)^{N-2}=I_{d}^{\prime}\left(v^{*}\right)$. To obtain the implied slopes at $w^{*}$, add $A 2+B 1+Y$ to the $L H S$ numerator and only $A 2+B 1$ to the $R H S$ 
numerator. These additions clearly preserve the inequality: $I_{c}^{\prime}\left(w^{*}\right)=\left(\frac{A 1+(A 2+B 1+Y)}{A+B+Y}\right)^{N-2}>\left(\frac{A 1+X+(A 2+B 1)}{A+B+X+Q}\right)^{N-2}=I_{d}^{\prime}\left(w^{*}\right)$. This is a contradiction with the necessary ordering of slopes for a second intersection at $w^{*}: I_{c}^{\prime}\left(w^{*}\right)<I_{d}^{\prime}\left(w^{*}\right)$.

Figure A1: Cases 1 and 2 of the $I\left(v_{2} \mid d\right)$ isobid in Proposition 5

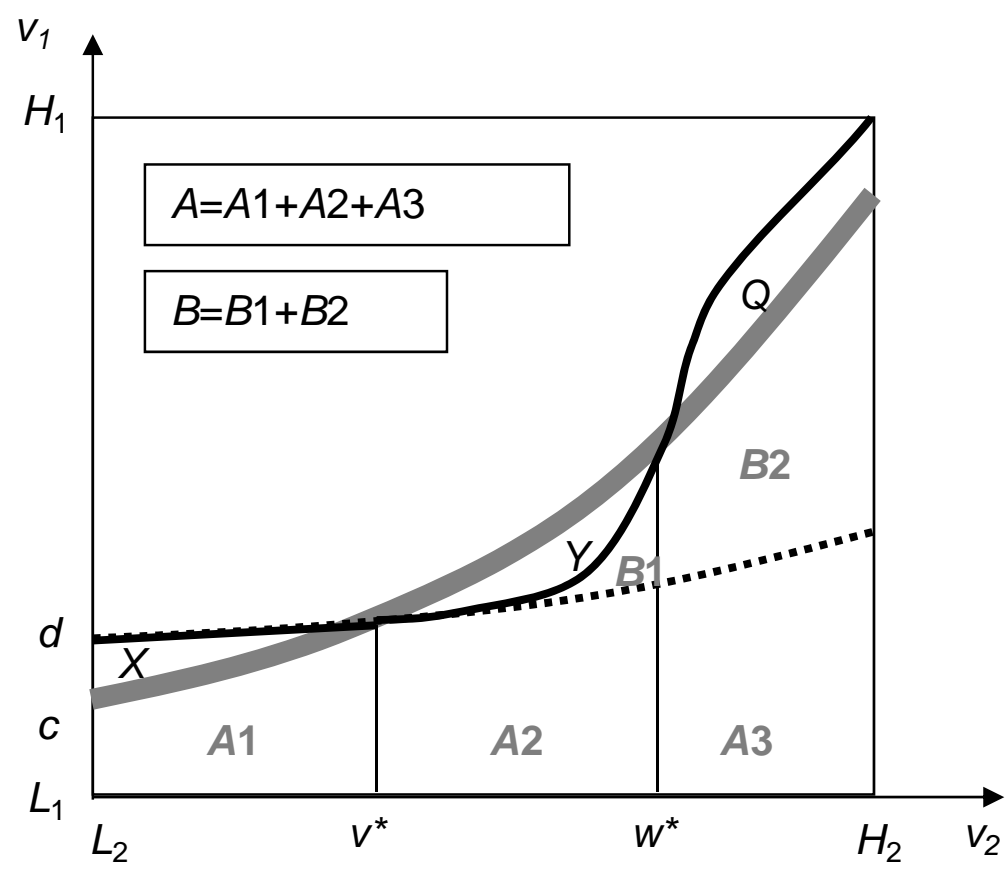

Note to Figure: The solid convex function is the $I\left(v_{2} \mid c\right)$ isobid. The dotted line is the Case 1 possibility for the $I\left(v_{2} \mid d\right)$ isobid, and the remainig solid line is Case 2. All marked areas denote probability mass between curves.

Case 3: The only remaining possibility that $v^{*}$ is in fact a point of tangency, namely $I_{c}^{\prime}\left(v^{*}\right)=I_{d}^{\prime}\left(v^{*}\right)$ and $I_{d}(v)>I_{c}(v)$ everywhere other than at $v^{*}$. Since Cases 1 and 2 rule out any intersections, tangency at $v^{*}$ means the tangency holds for all $z \in[c, d]$, and the curvatures of all the $I_{z}$ at $v^{*}$ must be nondecreasing in $z: \frac{d I_{z}^{\prime \prime}\left(v^{*}\right)}{d z} \geq 0$. Once again, equilibrium implies this ordering of curvatures cannot happen: Let the slope at $v^{*}$ be equal to some $\lambda$. Then, for every $z \in[c, d], \frac{F^{N-2}\left(I_{z}, v^{*}\right)}{F^{N-2}\left(I_{z}, H_{2}\right)}=G_{2}\left(v^{*} \mid z\right)=\lambda$, and so $I_{z}(v)=z+\lambda \int_{0}^{v} \frac{F^{N-2}\left(I_{z}, x\right)}{F^{N-2}\left(I_{z}, v^{*}\right)} d x$. Differentiate 
twice

$$
I_{z}^{\prime \prime}(v)=\frac{\lambda(N-2) F^{N-3}\left(I_{z}, v\right)}{F^{N-2}\left(I_{z}, v^{*}\right)} \int_{L_{1}}^{I_{z}(v)} f\left(v_{1}, v_{2}\right) d v_{1} \Rightarrow I_{z}^{\prime \prime}\left(v^{*}\right)=\frac{\lambda(N-2)}{F\left(I_{z}, v^{*}\right)} \int_{L_{1}}^{I_{z}\left(v^{*}\right)} f\left(v_{1}, v_{2}\right) d v_{1} .
$$

Since $I_{z}\left(v^{*}\right)$ is a constant for $z \in[c, d]$ and $F\left(I_{z}, v^{*}\right)$ strictly increases in $z$ because of full support, $I_{z}^{\prime \prime}\left(v^{*}\right)$ strictly decreases in $z$, a contradiction with $\frac{d I_{z}^{\prime \prime}\left(v^{*}\right)}{d z} \geq 0$. QED Proposition 5 .

Proof of Proposition 6: It is enough to show that $I\left(v_{2}, c_{1}\right)$ is upper semi-continuous at $c_{1}$, the proof of lower semi-continuity is analogous. Fix $c_{1}$ and consider any monotonically decreasing sequence of $\delta_{n}>0$ such that $\lim _{n \rightarrow \infty} \delta_{n}=0$. The corresponding sequence of isobids $\left\{I\left(v_{2} \mid c_{1}+\delta_{n}\right)\right\}_{n=1}^{\infty}$ is uniformly bounded because it projects into $\left[c_{1}, H_{1}+H_{2}-L_{2}\right]$, and it is equicontinuous because all isobids are nondecreasing and have slopes less than unity (1Lipschitz). Therefore, the Arzela-Ascoli Theorem implies that $\left\{I\left(v_{2} \mid c_{1}+\delta_{n}\right)\right\}_{n=1}^{\infty}$ has a uniformly convergent subsequence, and its limit is some 1-Lipschitz function $I_{+}\left(v_{2} \mid c_{1}\right)$. Monotonicity of the original sequence (Proposition 5) implies that it also converges uniformly to $I_{+}\left(v_{2} \mid c_{1}\right)$, because when $I\left(v_{2} \mid c_{1}+\delta_{n}\right)$ is an element of the convergent subsequence, all $I\left(v_{2} \mid c_{1}+\delta_{n+k}\right)$ are between $I\left(v_{2} \mid c_{1}+\delta_{n}\right)$ and $I_{+}\left(v_{2} \mid c_{1}\right)$ in the supremum metric $d(I, J)=\max _{v \in\left[L_{2}, H_{2}\right]}[|I(v)-J(v)|]$ : For every $\varepsilon>0$, a $\bar{\delta}>0$ exists such that for every $\delta<\bar{\delta}, d\left[I_{+}\left(\cdot \mid c_{1}\right), I\left(\cdot \mid c_{1}+\delta\right)\right]<\varepsilon$. Monotonicity further implies that for every $\delta, I\left(v_{2} \mid c_{1}\right) \leq I_{+}\left(v_{2} \mid c_{1}\right)<I\left(v_{2} \mid c_{1}+\delta\right)$.

That $I_{+}\left(v_{2} \mid c_{1}\right)=I\left(v_{2} \mid c_{1}\right)$ follows from $f$ having a bounded density $f<\bar{f}$ and from the uniqueness of isobids: The bounded density together with the uniform convergence of $\left\{I\left(v_{2} \mid c_{1}+\delta_{n}\right)\right\}_{n=1}^{\infty}$ implies that the implied sequence of distributions $\left\{G_{2}\left(c_{2} \mid c_{1}+\delta_{n}\right)\right\}_{n=1}^{\infty}$ also approaches the implied $G_{2}\left(c_{2} \mid I_{+}\left(c_{1}\right)\right)$ uniformly:

Pick any $\varepsilon>0$. Uniform convergence of isobids $\Rightarrow \exists \bar{\delta}$ be such that $\forall \delta<\bar{\delta}$, $\left|I\left(v_{2} \mid c_{1}+\delta\right)-I_{+}\left(v_{2} \mid c_{1}\right)\right|<\frac{F\left(c_{1}, H_{2}\right) \varepsilon}{\bar{f}\left(H_{2}-L_{2}\right)} . \forall \delta<\bar{\delta}$, this implies $\left|G_{2}\left(x \mid c_{1}+\delta\right)-G_{2}\left(x \mid I_{+}\right)\right|=$ 


$$
\begin{aligned}
& =\left|\left(\frac{F\left[I\left(\cdot \mid c_{1}+\delta\right), x\right]}{F\left[I\left(\cdot \mid c_{1}+\delta\right), H_{2}\right]}\right)^{N-2}-\left(\frac{F\left[I_{+}\left(\cdot \mid c_{1}\right), x\right]}{F\left[I_{+}\left(\cdot \mid c_{1}\right), H_{2}\right]}\right)^{N-2}\right|<\left|\frac{F\left[I\left(\cdot \mid c_{1}+\delta\right), x\right]}{F\left[I\left(\cdot \mid c_{1}+\delta\right), H_{2}\right]}-\frac{F\left[I_{+}\left(\cdot \mid c_{1}\right), x\right]}{F\left[I_{+}\left(\cdot \mid c_{1}\right), H_{2}\right]}\right|<
\end{aligned}
$$

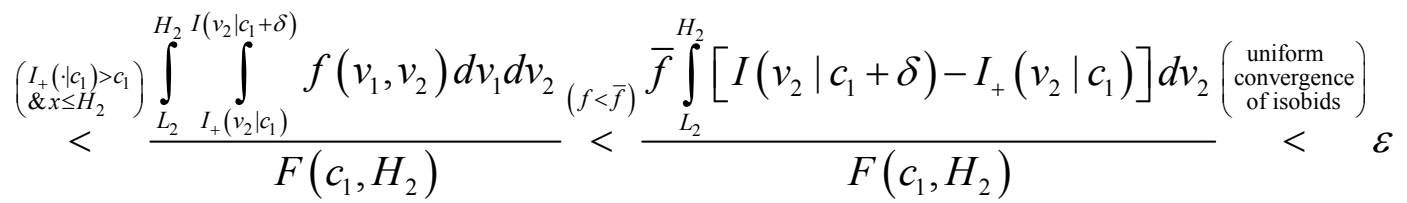

The uniform convergence of $\left\{G_{2}\left(c_{2} \mid c_{1}+\delta_{n}\right)\right\}_{n=1}^{\infty}$ in turn implies that the equilibrium relation from equation (2) $I\left(v_{2} \mid c_{1}+\delta\right)=\boldsymbol{T}\left[I\left(v_{2} \mid c_{1}+\delta\right) \mid c_{1}+\delta\right]$, which holds for every member of the sequence, is preserved in the limit.

$$
\begin{aligned}
I_{+}\left(v_{2} \mid c_{1}\right) & =\lim _{\delta \rightarrow 0} I\left(L_{2} \mid c_{1}+\delta\right)+\lim _{\delta \rightarrow 0} \int_{0}^{v_{2}} G_{2}^{N-2}\left(x \mid c_{1}+\delta\right) d x= \\
& =c_{1}+\int_{0}^{v_{2}} \lim _{\delta \rightarrow 0} G_{2}^{N-2}\left(x \mid c_{1}+\delta\right) d x=\boldsymbol{T}\left[I_{+}\left(v_{2} \mid c_{1}\right) \mid c_{1}\right]
\end{aligned}
$$

Since $I_{+}\left(v_{2} \mid c_{1}\right)=\boldsymbol{T}\left[I_{+}\left(v_{2} \mid c_{1}\right) \mid c_{1}\right]$, it must be true that $I_{+}\left(v_{2} \mid c_{1}\right)=I\left(v_{2} \mid c_{1}\right)$ because each isobid is unique (Proposition 3). QED Proposition 6.

Proof of Proposition 7: By the same arguments used in the proof of Proposition 6, any monotonic sequence $\left\{I\left(v_{2} \mid L_{1}+\delta_{n}\right)\right\}_{n=1}^{\infty}$ converges uniformly to some 1-Lipschitz function $J\left(v_{2}\right)$ such that $L_{1} \leq J\left(v_{2}\right)<I\left(v_{2} \mid L_{1}+\delta\right)$ and $J\left(L_{2}\right)=L_{1}$. This proof shows that $J\left(v_{2}\right)$ must be the constant function $J\left(v_{2}\right)=L_{1}$. Suppose $J\left(v_{2}\right)>L_{1}$ for some $v_{2}>L_{2}$, and so there is a positive mass under $J: F\left(J, H_{2}\right)>0$ (from continuity of $J$ together with full support of $f$ ). $F\left(J, H_{2}\right)>0$ in turn implies (see proof of Proposition 6) that the RHS equation (2) is welldefined, and so $J=\boldsymbol{T}\left(J \mid L_{1}\right)$, that is, that $J$ is a valid isobid for the bid-level $L_{1}$. The rest of this proof shows no $J:\left[L_{2}, H_{2}\right] \rightarrow\left[L_{1}, L_{1}+H_{2}-L_{2}\right]$ can exist such that $F\left(J, H_{2}\right)>0$, and $J=\boldsymbol{T}\left(J \mid L_{1}\right)$. The following Lemma 2 is crucial for proving the impossibility:

Lemma 2: Suppose $F\left(J, H_{2}\right)>A>0$ and $0<f<\bar{f}$. Then, $\forall \alpha \in R_{+} \&$ integer $M \geq 0$ :

$$
J\left(v_{2}\right) \leq L_{1}+\alpha \frac{v_{2}^{M}}{M !} \Rightarrow \boldsymbol{T}\left(J \mid L_{1}\right)\left(v_{2}\right)<L_{1}+\left(\frac{\alpha \bar{f}}{A}\right) \frac{v_{2}^{M+2}}{(M+2) !}
$$


Proof of Lemma $2: \boldsymbol{T}\left(J \mid L_{1}\right)\left(v_{2}\right)=L_{1}+\frac{\int_{L_{2}}^{v_{2}} \int_{L_{2}}^{z} \int_{L_{1}}^{J(w)} f\left(v_{1}, w\right) d v_{1} d w d z}{F\left(J, H_{2}\right)}\left(\begin{array}{l}F\left(J, H_{2}\right)>A \\ J\left(v_{2}\right) \leq L_{1}+\alpha \frac{v_{2}^{M}}{M !}\end{array}\right)$ $<L_{1}+\frac{\int_{L_{2}}^{v_{2}} \int_{L_{2}}^{z} \int_{L_{1}}^{L_{1}+\alpha \frac{w^{M}}{M !}} f\left(v_{1}, w\right) d v_{1} d w d z}{A} \stackrel{\left(\begin{array}{c}f<\bar{f} \\ L_{2} \geq 0\end{array}\right)}{<} L_{1}+\frac{\alpha \bar{f}}{A} \int_{0}^{v_{2}} \int_{0}^{z} \frac{w^{M}}{M !} d w d z=L_{1}+\left(\frac{\alpha \bar{f}}{A}\right) \frac{v_{2}^{M+2}}{(M+2) !}$

Lemma 2 implies successive applications of $\boldsymbol{T}\left(\cdot \mid L_{1}\right)$ must eventually contract $J$ arbitrarily close to $L_{1}$ - a contradiction with a fixed point of $\boldsymbol{T}\left(\cdot \mid L_{1}\right)$ satisfying $F\left(J, H_{2}\right)>A>0$ : From $J\left(v_{2}\right)$ being a 1-Lipschitz function, $J\left(v_{2}\right) \leq L_{1}+v_{2}$. Therefore,

$$
\begin{aligned}
& J\left(v_{2}\right) \leq L_{1}+v_{2} \Rightarrow \boldsymbol{T}\left(J \mid L_{1}\right)\left(v_{2}\right)<L_{1}+\left(\frac{\bar{f}}{A}\right) \frac{v_{2}^{3}}{3 !} \Rightarrow \boldsymbol{T}^{2}\left(J \mid L_{1}\right)\left(v_{2}\right)<L_{1}+\left(\frac{\bar{f}}{A}\right)^{2} \frac{v_{2}^{5}}{5 !} \ldots \\
& \stackrel{(\text { induction) }}{\Rightarrow} \boldsymbol{T}^{K}\left(J \mid L_{1}\right)\left(v_{2}\right)<L_{1}+\left(\frac{\bar{f}}{A}\right)^{K} \frac{v_{2}^{2 K+1}}{(2 K+1) !} \Rightarrow \forall v_{2} \in\left[L_{2}, H_{2}\right]: \lim _{K \rightarrow \infty} \boldsymbol{T}^{K}\left(J \mid L_{1}\right)\left(v_{2}\right)=0
\end{aligned}
$$

Note that a crucial difference exists between Lemmas 1 and 2: Lemma 1 shows that for $c_{1}>L_{1}$, enough repetitions of $\boldsymbol{T}\left(\cdot \mid c_{1}\right)$ eventually contract the images of two_candidate functions $\boldsymbol{T}^{K}\left(I_{1} \mid c_{1}\right)$ and $\boldsymbol{T}^{K}\left(I_{2} \mid c_{1}\right)$ to be closer to each other (in the supremum metric) than $I_{1}$ and $I_{2}$. In contrast, Lemma 2 shows that for $c_{1}=L_{1}$, enough repetitions of $\boldsymbol{T}\left(\cdot \mid c_{1}\right)$ eventually contract the image of any single candidate function $I_{1}$ to be arbitrarily close to $L_{1}$. However, $L_{1}$ is not a fixed point of $\boldsymbol{T}\left(\cdot \mid L_{1}\right)$ because $\boldsymbol{T}\left(L_{1} \mid L_{1}\right)$ is not defined: $\boldsymbol{T}\left(\cdot \mid L_{1}\right)$ is not a continuous function on the space of 1-Lipschitz functions $J:\left[L_{2}, H_{2}\right] \rightarrow\left[L_{1}, L_{1}+H_{2}-L_{2}\right] . \quad$ QED Proposition 7

Proof of Proposition 8: The objective function of a bidder with valuations $\left(v_{1}, v_{2}\right)$ and an expected surplus function $S\left(v_{2}, c_{1}\right)$ is $\Pi\left(\beta \mid v_{1}, v_{2}, S\right)=\int_{0}^{\beta}\left(v_{1}-c_{1}\right) d G_{1}\left(c_{1}\right)+\int_{\beta}^{\infty} S\left(v_{2}, c_{1}\right) d G_{1}\left(c_{1}\right)$. The first-order conditions are $F O C: \frac{d \Pi}{d \beta}=v_{1}-\beta-S\left(v_{2}, \beta\right)$. The function $\Pi\left(\beta \mid v_{1}, v_{2}, S\right)$ is concave at the FOC when it does not decrease in $c_{1}$ faster than unity. When $S$ is partially 
differentiable, the $S O C$ is: $\left.\frac{d^{2} \Pi}{d \beta^{2}}\right|_{F O C} \propto-1-\frac{\partial S}{\partial \beta}\left(v_{2}, \beta\right)<0 \Leftrightarrow \frac{\partial S}{\partial \beta}\left(v_{2}, \beta\right)>-1$. When $S$ is not partially differentiable, $S\left(v_{2}, d\right)-S\left(v_{2}, c\right)>(-1)(d-c)$ is sufficient for concavity of $\Pi\left(\beta \mid v_{1}, v_{2}, S\right)$ at the $F O C$. While $\Pi$ may not be globally concave, the $F O C$ characterizes the global optimum of $\Pi$ because $v_{1}-\beta>S\left(v_{2}, \beta\right)$ iff $\beta<\beta_{F O C}$. This single-crossing property is again implied by regularity, as discussed next.

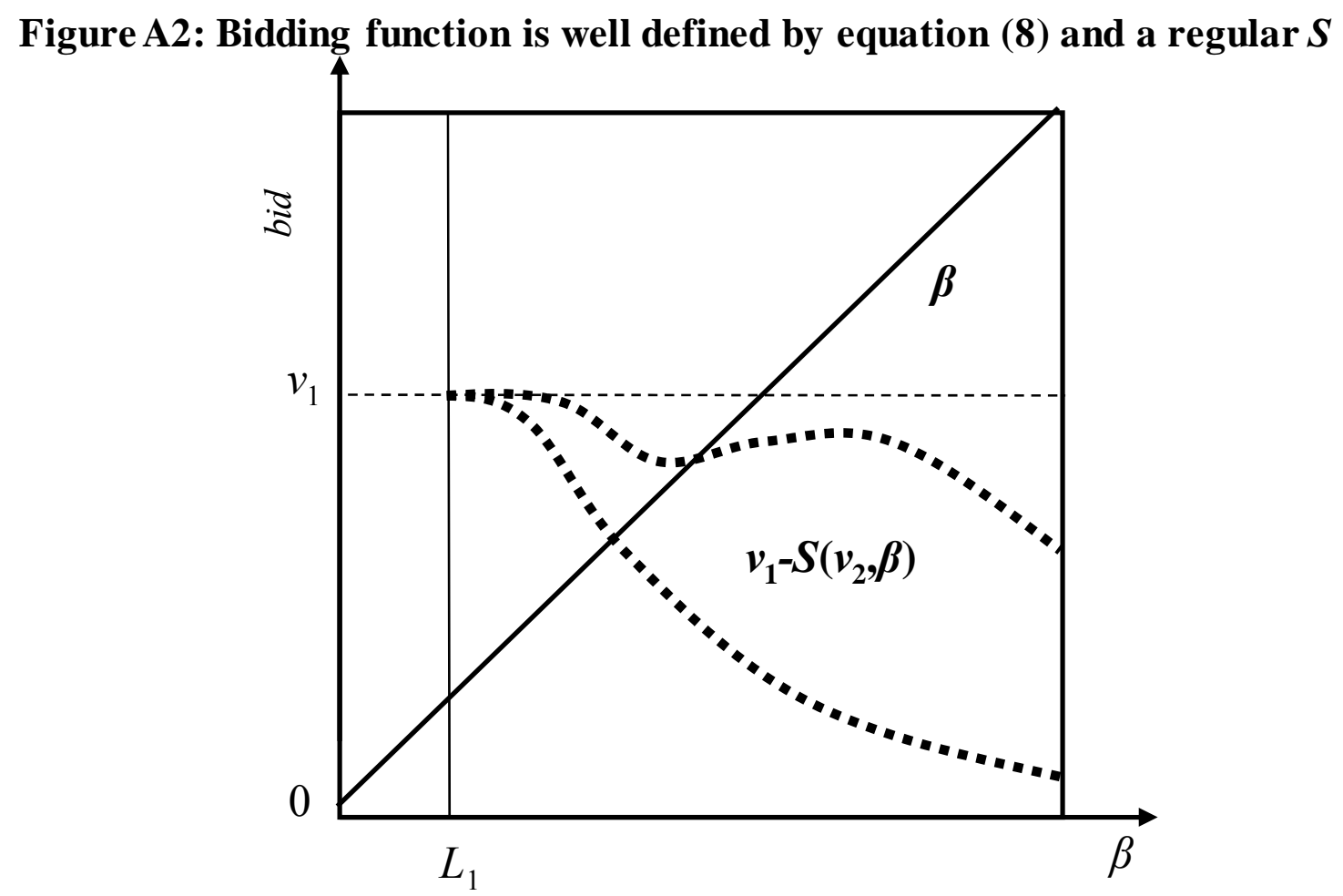

Note to Figure: The solid diagonal line is the LHS of equation (8). The two dashed lines illustrate two possible RHS of equation (8): the lower dashed line corresponds to $S$ increasing in bid-level; the upper dashed line corresponds to $S$ locally decreasing in bid-level, but slower than unity.

To see that the $b_{1}\left(v_{1}, v_{2}\right)$ is well defined by the FOC, consider the following implications of regularity (and see Figure A1 for an illustration): Part A) implies that the RHS of equation (8) does not increase in $\beta$ faster than unity. Part $C$ ) fixes the intercept of the RHS at $v_{1}-S\left(v_{2}, L_{1}\right)=v_{1} \geq L_{1}$. Since the LHS of equation (8) is an identity function, continuity of $S$ in $c_{1}$ (part B of regularity) implies (via the Intermediate Value Theorem) that the RHS must eventually intersect the LHS at some $\beta \geq L_{1}$ that solves equation (8). Such a solution is unique because the 
slope of $v_{1}-S\left(v_{2}, \beta\right)$ in $\beta$ is less than unity everywhere, and so a second intersection is impossible.

The single crossing property outlined above also shows that abstaining from the first auction cannot be a profitable deviation. Suppose $N-1$ competitors bid according to $b_{1}(\cdot)$, and a focal bidder deviates to an abstention instead of bidding $B \equiv b_{1}\left(v_{1}, v_{2}\right)$. The only way such a deviation can make any difference in the outcome of the game is if the focal bidder would have won the first auction, i.e., if $B>c_{1}$. For $B>c_{1}$, the deviation thus yields the expected secondstage surplus of $\int_{0}^{B} S\left(v_{2}, c_{1}\right) d G_{1}\left(c_{1}\right)$ instead of $\int_{0}^{B}\left(v_{1}-c_{1}\right) d G_{1}\left(c_{1}\right)$ available from bidding $B$. But the profit from the deviation must be lower, because $v_{1}-\beta>S\left(v_{2}, \beta\right)$ for all $\beta<B$. QED Proposition 8.

Proof of Theorem 1: Proposition 3 shows that the (symmetric pure-strategy Nash) equilibrium conditions imply the existence of a unique isobid function for each bid-level $c_{1} \in\left(L_{1}, H_{1}\right]$. These equilibrium isobids in turn imply a unique expected surplus function $S\left(v_{2}, c_{1}\right)=I\left(v_{2} \mid c_{1}\right)-c_{1}$ defined on $\left[L_{2}, H_{2}\right] \times\left(L_{1}, H_{1}\right]$. The rest of the Propositions (4-7) then show that the equilibrium $S\left(v_{2}, c_{1}\right)$ is regular on the closure of $\left[L_{2}, H_{2}\right] \times\left(L_{1}, H_{1}\right]$. Since the candidates for equilibrium isobids on $c_{1} \in\left(L_{1}, H_{1}\right]$ thus imply a unique regular surplus function $S\left(v_{2}, c_{1}\right)$ on $\left[L_{2}, H_{2}\right] \times\left[L_{1}, H_{1}\right]$, Proposition 8 implies a unique symmetric pure-strategy bidding function $b_{1}\left(v_{1}, v_{2}\right)=v_{1}-S\left(v_{2}, b_{1}\left(v_{1}, v_{2}\right)\right)$ exists that is a best response to itself in the sense of equation (2). Properties of $b_{1}$ : First, $b_{1}\left(L_{1}, v_{2}\right)=L_{1}$ because part C of regularity implies $L_{1}=L_{1}-S\left(v_{2}, L_{1}\right)$. Second, Proposition 4 implies $b_{1}\left(v_{1}, L_{2}\right)=v_{1}$ and $v_{1}>b_{1}\left(v_{1}, v_{2}\right)>L_{1}$ away from $\left(L_{1}, L_{2}\right)$. Third, regularity and Proposition 4 imply the comparative statics in valuations: suppose for simplicity that $S$ is partially differentiable in $c_{1}$, and implicitly differentiate of the first-order condition (2): $\frac{\partial b_{1}}{\partial v_{2}}=-\frac{\partial S}{\partial v_{2}} /\left(1+\left.\frac{\partial S}{\partial c_{1}}\right|_{c_{1}=b_{1}}\right)<0$ away from $\left(L_{1}, L_{2}\right)$ because the numerator is negative by 
Proposition 4 (and the denominator is positive by part A). Similarly, $\frac{\partial b_{1}}{\partial v_{1}}=\left(1+\left.\frac{\partial S}{\partial c_{1}}\right|_{c_{1}=b_{1}}\right)^{-1}>0$, so $b_{1}\left(v_{1}, v_{2}\right)$ is increasing in $v_{1}$ but not necessarily at a constant speed equal to unity. This confirms that the equilibrium is strictly monotone. The curvature of $b_{1}\left(v_{1}, v_{2}\right)$ is ambiguous. Finally, the comparative static in the number of bidders arises because $S\left(v_{2}, c\right)<E\left(v_{2}\right)$, where $E\left(v_{2}\right)$ is the expected surplus from facing (in a second-price sealed-bid auction) $N$-2 bidders drawn randomly from the $N-1$ lowest bidders of the original $N$ bidders. Let the valuations of the $N-1$ lowest bidders of the original $N$ bidders have a distribution function $\tilde{G}$. Then, $E\left(v_{2}\right)$ is just $E\left(v_{2}\right)=\int_{L_{2}}^{v_{2}} \tilde{G}^{N-2}(x) d x$, which follows from integration by parts as in equation (3). The integrand converges to zero uniformly as $N$ approaches infinity as long as $\tilde{G}\left(v_{2}\right)<1$. From full support of $f$, this holds for all $v_{2}<H_{2}$. Since $\tilde{G}^{N-2}(x)$ approaches zero on $\left[L_{2}, H_{2}\right)$ pointwise, its integral $E\left(v_{2}\right)$ must approach zero even $v_{2}=H_{2}$. QED Theorem 1.

Proof of Proposition 9: The equilibrium isobids can be simply scaled up by while preserving the equilibrium relation in equation (2). Specifically, when $I\left(v_{2} \mid c_{1}\right)$ is the equilibrium isobid on $\left[L_{1}, H_{1}\right] \times\left[L_{2}, H_{2}\right]$, then $I_{w}\left(w_{2} \mid A+B c_{1}\right)=A+B I\left(\frac{w_{2}-A}{B} \mid c_{1}\right)$ is the equilibrium isobid of the valuations transformed to $\left[A+B L_{1}, A+B H_{1}\right] \times\left[A+B L_{2}, A+B H_{2}\right]$ support. The key to the result is that the conditional probability under the transformed $I$ remains unchanged:

$$
\begin{aligned}
& F_{w}\left[A+B I\left(\frac{w_{2}-A}{B} \mid c_{1}\right), A+B x\right]=\int_{A+B L_{2}}^{(d e f)} \int_{A+B L_{1}}^{A+B x} \frac{1}{B^{2}} f\left(\frac{w_{1}-A}{B}, \frac{w_{2}-A}{B}\right) d w_{1} d w_{2}= \\
& \stackrel{\left(v_{i}=\frac{w_{i}-A}{B}\right)}{=} \int_{L_{2}}^{x} \int_{L_{1}}^{I\left(v_{2} \mid c_{1}\right)} f\left(v_{1}, v_{2}\right) d v_{1} d v_{2}=F\left[I\left(\cdot \mid c_{1}\right), x\right] \text { where } f_{w}\left(w_{1}, w_{2}\right)=\frac{1}{B^{2}} f\left(\frac{w_{1}-A}{B}, \frac{w_{2}-A}{B}\right)
\end{aligned}
$$

Therefore, $I_{w}$ is the fixed point of the appropriately defined $\boldsymbol{T}$ from equation (2): 


$$
\begin{aligned}
& \boldsymbol{T}_{w}\left[I_{w} \mid A+B c_{1}\right]\left(w_{2}\right)=A+B c_{1}+\int_{A+B L_{2}}^{w_{2}}\left(\frac{F_{w}\left[A+B I\left(\frac{w_{2}-A}{B} \mid c_{1}\right), z\right]}{F_{w}\left[A+B I\left(\frac{w_{2}-A}{B} \mid c_{1}\right), A+B H_{2}\right]}\right)^{N-2} d z= \\
& \quad\left(x=\frac{z-A}{B}\right) \\
& \quad=A+B c_{1}+B \int_{L_{2}}^{\frac{w_{2}-A}{B}}\left(\frac{F\left(I\left(\cdot \mid c_{1}\right), x\right)}{F\left(I\left(\cdot \mid c_{1}\right), 1\right)}\right)^{N-2} d x=A+B I\left(\frac{w_{2}-A}{B} \mid c_{1}\right)=I_{w}\left(w_{2} \mid A+B c_{1}\right)
\end{aligned}
$$

The equilibrium surplus function is therefore $S_{w}\left(w_{2}, A+B c_{1}\right)=B S\left(\frac{w_{2}-A}{B}, c_{1}\right)$, and when $\beta$ is a solution to $\beta=v_{1}-S\left(\frac{w_{2}-A}{B}, \beta\right)$, then multiplying both sides of the equation by $B$ and adding $A$ leads to $A+B \beta=A+B v_{1}-B S\left(\frac{w_{2}-A}{B}, \beta\right)=w_{1}-B S_{w}\left(w_{2}, A+B \beta\right)$, so $A+B \beta$ is a solution to $\gamma=w_{1}-B S_{w}\left(w_{2}, \gamma\right) \cdot Q E D$ Proposition 9

\section{Example: Three bidders, $\boldsymbol{f}$ uniform on $[0,1] \times[0, K]$}

The uniform distribution simplifies the integral under an isobid to: $\int_{0}^{I\left(v_{2} \mid c_{1}\right)} f\left(v_{1}, v_{2}\right) d v_{1}=\frac{\min \left[I\left(v_{2} \mid c_{1}\right), 1\right]}{K}$. Three bidders simplify the equilibrium condition (2') because the crucial exponent is $N-2=1$. There are two cases depending on whether $I\left(K \mid c_{1}\right)<1$ (the upper bound of $v_{1}$ ) or not.

Case 1: $c_{1}$ low-enough that $I\left(K \mid c_{1}\right)<1$ : Suppressing the conditioning on $c_{1}$ for clarity, equation (2') becomes: $I^{\prime \prime}(v) \int_{0}^{K} I(x) d x=I(v)$ with initial conditions $I(0)=c_{1}, I^{\prime}(0)=0$. This differential-integral equation has a unique solution for every $c_{1}$ obtained as follows: first, solve a simpler differential equation $I^{\prime \prime}(v)\left(1 / A^{2}\right)=I(v)$ with $I(0)=c_{1}, I^{\prime}(0)=0$ for an arbitrary constant $A$. This simpler differential equation has a unique solution of $I(v)=c_{1} \cosh (A v)$ which integrates to $\int_{0}^{K} I(v) d v=\frac{c_{1} \sinh (K A)}{A}$. Therefore, the solution to (2') can be characterized as 
$I(v)=c_{1} \cosh \left[A\left(c_{1}\right) v\right]$ where the function $A\left(c_{1}\right)$ satisfies $1=c_{1} A\left(c_{1}\right) \sinh \left(K A\left(c_{1}\right)\right)$. The implicitly defined function $A\left(c_{1}\right)$ exists because $A \sinh (K A)$ is continuous, monotonically increasing on $[0, \infty)$, zero at $A=0$, and rising without bound as $A$ approaches infinity.

Case 2: $c_{1}$ high-enough that $c_{1} \cosh \left[A\left(c_{1}\right) K\right]>1$ for the above-defined function $A\left(c_{1}\right)$. Fix $c_{1}$ again and suppress conditioning on it from notation. There is now a point $L_{2}<v^{*}<H_{2}$ such that $I\left(v^{*}\right)=1$, and the differential-integral equation (2') needs to acknowledge the support of $f$ in $F^{N-2}\left(I, H_{2}\right)$. For $v_{2}<v^{*}$, the equation is:

$$
I^{\prime \prime}\left(v_{2}\right)\left[\frac{1}{K} \int_{0}^{v^{*}} I\left(v_{2}\right) d v_{2}+\left(1-\frac{v^{*}}{K}\right)\right]=\frac{I\left(v_{2}\right)}{K} \Leftrightarrow I^{\prime \prime}\left(v_{2}\right)\left[\int_{0}^{v^{*}} I\left(v_{2}\right) d v_{2}+K-v^{*}\right]=I\left(v_{2}\right) \quad \text { with initial }
$$

conditions $I(0)=c_{1}, I^{\prime}(0)=0$. Arguments analogous to Case 1 imply that $I\left(v_{2}\right)=c_{1} \cosh \left(B v_{2}\right)$ for some constant $B$ such that $\int_{0}^{v^{*}} I\left(v_{2}\right) d v_{2}+K-v^{*}=\frac{1}{B^{2}}$. The cutoff value is $v^{*}=\frac{\operatorname{arcsech}\left(c_{1}\right)}{B}$. The integral of $I 0$ to $v^{*}$ simplifies to $\int_{0}^{v^{*}} I\left(v_{2}\right) d v_{2}=\frac{c_{1}}{B} \int_{0}^{\operatorname{arcsech}\left(c_{1}\right)} \cosh (z) d z=\frac{\sqrt{1-c_{1}^{2}}}{B}$. To find the constant $B$, I need to make sure that $K-\frac{\operatorname{arcsech}\left(c_{1}\right)-\sqrt{1-c_{1}^{2}}}{B}=\frac{1}{B^{2}}$, which transforms to $K B-\frac{1}{B}=\log \left(1+\sqrt{1-c_{1}^{2}}\right)-\log \left(c_{1}\right)-\sqrt{1-c_{1}^{2}}$. The implicitly defined function $B\left(c_{1}\right)$ exists because the RHS is positive, setting $B=1 / \sqrt{K}$ makes LHS=0 and the LHS then increases without bound for $B>1 / \sqrt{K}$. Therefore, there is a unique $B$ such that LHS=RHS. 


\section{References}

Black, Jane, and David de Meza. 1992. Systematic Price Differences between Successive Auctions are no Anomaly. Journal of Economics and Management Strategy 1 (4): 607-8.

Budish, Eric. 2008. Sequencing and Information Revelation in Auctions for Imperfect Market Design: Understanding eBay's Market Design. Working paper. Harvard University.

Burguet, Roberto, 2005. The Condominium Problem: Auctions for Substitutes. Review of Economic Design 9: 73-90.

Che, Yeon-Koo, and Ian Gale. 1998. Standard Auctions with Financially Constrained Bidders. Review of Economic Studies 65 (1): 1-21.

Engelbrecht-Wiggans, Richard. 1994. Sequential Auctions of Stochastically Equivalent Objects. Economic Letters 44: 87-90.

Gale, Ian L., and Donald B. Hausch. 1994. Bottom-Fishing and Declining Prices in Sequential Auctions. Games and Economic Behavior 7: 318-31.

Haile, Philip A. 2000. Partial Pooling at the Reserve Price in Auctions with Resale Opportunities. Games and Economic Behavior 33(2): 231-248

Holt, Charles A. and Roger Sherman. 1994. The Loser's Curse. American Economic Review 84(3): 642-52.

Jehiel, Philippe and Benny Moldovanu and Ennio Stacchetti. 1999. Multidimensional Mechanism Design for Auctions with Externalities. Journal of Economic Theory 85(2): 258293.

Jehiel, Philippe and Benny Moldovanu. 2000. Auctions with downstream interaction among buyers, RAND Journal of Economics 31 : 768-791.

Jofre-Bonet, Mireia, and Martin Pesendorfer. 2003. Estimation of a Dynamic Auction Game. Econometrica 71: 1443-89. 
Katzman, Brett. 1999. A Two-Stage Sequential Auction with Multi-Unit Demands. Journal of Economic Theory 86: 77-99.

Krishna, Vijay. 2002. Auction Theory. Academic Press of Elsevier Science, London, UK.

Milgrom, Paul R., and Robert Weber. 2000. A Theory of Auctions and Competitive Bidding II. Working paper in 1982, now published in: Klemperer, P. (ed.), The Economic Theory of Auctions. Cheltenham, UK: Paul, Edward Elgar Publishing.

Maskin, Eric and Riley, John. 1989. Optimal Multi-unit Auctions, in Frack Hahn (Ed.), The Economics of Missing Markets, Information, and Games. Oxford University Press, UK.

Myerson, Roger. 1981. Optimal Auction Design. Mathematics of Operations Research 6: 58-73.

Pesendorfer, Wolfgang and Jeroen M. Swinkels. 1997. The Loser's Curse and Information Aggregation in Common Value Auctions. Econometrica 65(6) : 1247-1281

Reiß, J. Philipp and Schöndube, Jens Robert. 2008. First-price equilibrium and revenue equivalence in a sequential procurement auction model. Economic Theory, forthcoming.

Vickrey, William. 1961. Counterspeculation, Auctions, and Competitive Sealed Tenders. Journal of Finance 16(1): 8-37.

Zeithammer, Robert., 2006. Forward-Looking Bidding in Online Auctions. Journal of Marketing Research 43(3): 462-76. 